\title{
HIGHER-ORDER ALEXANDER INVARIANTS AND FILTRATIONS OF THE KNOT CONCORDANCE GROUP
}

\author{
TIM D. COCHRAN AND TAEHEE KIM
}

\begin{abstract}
We establish certain "nontriviality" results for several filtrations of the smooth and topological knot concordance groups. First, as regards the $n$-solvable filtration of the topological knot concordance group, $\mathcal{C}$, defined by K. Orr, P. Teichner and the first author:

$$
0 \subset \cdots \subset \mathcal{F}_{(n .5)} \subset \mathcal{F}_{(n)} \subset \cdots \subset \mathcal{F}_{(1.5)} \subset \mathcal{F}_{(1.0)} \subset \mathcal{F}_{(0.5)} \subset \mathcal{F}_{(0)} \subset \mathcal{C},
$$

we refine the recent nontriviality results of Cochran and Teichner by including information on the Alexander modules. These results also extend those of C. Livingston and the second author. We exhibit similar structure in the closely related symmetric Grope filtration of $\mathcal{C}$. We also show that the Grope filtration of the smooth concordance group is nontrivial using examples that cannot be distinguished by the Ozsváth-Szabó $\tau$-invariant nor by J. Rasmussen's $s$ invariant. Our broader contribution is to establish, in "the relative case", the key homological results whose analogues Cochran-Orr-Teichner established in "the absolute case".

We say two knots $K_{0}$ and $K_{1}$ are concordant modulo $n$-solvability if $K_{0} \#\left(-K_{1}\right) \in \mathcal{F}_{(n)}$. Our main result is that, for any knot $K$ whose classical Alexander polynomial has degree greater than 2, and for any positive integer $n$, there exist infinitely many knots $K_{i}$ that are concordant to $K$ modulo $n$-solvability, but are all distinct modulo n.5-solvability. Moreover, the $K_{i}$ and $K$ share the same classical Seifert matrix and Alexander module as well as sharing the same higher-order Alexander modules and Seifert presentations up to order $n-1$.
\end{abstract}

\section{INTRODUCTION}

Oriented knots $K_{0}$ and $K_{1}$ in the 3 -sphere are called (topologically) concordant if there is a topologically locally flat proper embedding of $S^{1} \times[0,1]$ into $S^{3} \times[0,1]$ that restricts to the $K_{i}$ on $S^{3} \times\{i\}$. Equivalently, $K_{0}$ is concordant to $K_{1}$ if $K_{0} \#\left(-K_{1}\right)$ is a (topologically) slice knot, that is, if $K_{0} \#\left(-K_{1}\right)$ bounds a topologically locally flat disk in the 4-ball. Here ' $\#$ ' denotes the connected sum, and $-K_{1}$ is the mirror image of $K_{1}$ with reversed string orientation. Concordance is an equivalence relation on the set of oriented knots, and the set of equivalence classes (concordance classes) forms an abelian group, $\mathcal{C}$, under the operation of connected sum. This group is called the (topological) knot concordance group. The classification of the knot concordance group is still open, and it has been one of the central problems in knot theory. A common strategy of investigating the knot concordance group is to extract information from abelian covers or metabelian covers of the exterior of a

Received by the editors January 26, 2005 and, in revised form, October 21, 2005.

2000 Mathematics Subject Classification. Primary 57M25.

The first author was partially supported by the National Science Foundation. 
knot (see [L], [CS], [CG], [G], [KL], [Le], [Fr]). On the other hand, requiring smooth embedding instead of topologically locally flat embedding, we can define the smooth knot concordance group, which is denoted by $\mathcal{C}^{\text {smooth }}$. In smooth knot concordance theory, there have been recent and very interesting developments using knot Floer homology [OS] and Khovanov homology [Ra]. In this paper we concern ourselves mainly with topological knot concordance. Hence we work in the topologically locally flat category unless mentioned otherwise.

Cochran, Orr, and Teichner (henceforth COT) recently made significant progress in the study of $\mathcal{C}$ using derived covers of the exterior of a knot [COT1]. (A derived cover is a covering space corresponding to a derived subgroup of the fundamental group of the space.) In particular, they defined a filtration $\left\{\mathcal{F}_{(n)}\right\}_{n \in \frac{1}{2} \mathbb{N}_{0}}$ of the knot concordance group:

$$
0 \subset \cdots \subset \mathcal{F}_{(n .5)} \subset \mathcal{F}_{(n)} \subset \cdots \subset \mathcal{F}_{(1.5)} \subset \mathcal{F}_{(1.0)} \subset \mathcal{F}_{(0.5)} \subset \mathcal{F}_{(0)} \subset \mathcal{C}
$$

Here $\mathcal{F}_{(n)}$ is a subgroup of $\mathcal{C}$ consisting of $n$-solvable knots. (A knot $K$ is said to be $n$-solvable if the zero surgery on the knot in the 3 -sphere bounds a spin 4 -manifold $W$ which satisfies certain conditions on integral homology groups and intersection forms on the $n$-th derived cover of $W$. In this case, we say $K$ is $n$-solvable via $W$, and $W$ is called an $n$-solution for $K$. Refer to [COT1, Section 8].) COT showed that the previously known abelian and metabelian concordance invariants are reflected at the first stage of their filtration [COT1], implying that the abelian group $\mathcal{F}_{(1.0)} / \mathcal{F}_{(1.5)}$ has infinite rank. They also established that $\mathcal{F}_{(2.0)} / \mathcal{F}_{(2.5)}$ has infinite rank [COT2]. Moreover, Cochran and Teichner showed that $\mathcal{F}_{(n)} / \mathcal{F}_{(n .5)}$ has positive rank for every integer $n \geq 2[\mathrm{CT}]$. It is still unknown whether or not $\mathcal{F}_{(n)} / \mathcal{F}_{(n .5)}$ is infinitely generated for $n>2$, or whether or not $\mathcal{F}_{(n .5)} / \mathcal{F}_{(n+1)}$ is nontrivial. Thus, although $\mathcal{F}$ has been shown to be highly nontrivial, many questions about its structure remain open.

These non-triviality results have been refined in the case $n=1$. C. Livingston recently asked if one can always find non-concordant knots which share a given (classical) Seifert form. That is, given $K$, can one always find other knots, distinct up to concordance, which share the same (classical) Seifert form as $K$ ? As he observed, if $K$ has the Seifert form of an unknot (has Alexander polynomial 1), then the answer is certainly "No", since all knots with Alexander polynomial 1 are topologically concordant to the trivial knot and hence concordant to each other (by M. Freedman's work $[\mathrm{F}],[\mathrm{FQ}])$. Livingston gave a partial answer in the positive to his question using Casson-Gordon invariants [Li]. This work was completed by the second author, who showed that there always exist such knots for a given (classical) Seifert form if and only if the Alexander polynomial of the Seifert form is not trivial. More precisely, in $[\mathrm{K}]$ he showed that for a given knot $K$ whose Alexander polynomial has degree at least 2, there are infinitely many knots $K_{i}$ (with $K_{0}=K$ ) such that $K_{i}-K$ is 1 -solvable, but $K_{i}-K_{j}(i \neq j)$ is not (1.5)solvable, and $K_{i}$ shares the same (classical) Seifert form (hence the same (classical) Alexander module) as the knot $K$. We view these results as non-triviality results for $\mathcal{F}$ that are finer (for $n=1$ ) than those of [COT1], [COT2], and [CT]. They suggest further questions. Is the Livingston-Kim result true for $n>1$ ? Moreover, is there an even finer result that constructs such examples while fixing not only the (classical) Seifert form (hence the (classical) Alexander module) but also the 
higher-order Seifert forms and higher-order Alexander modules developed in [C]? This paper answers these questions in the positive as long as the degree of the Alexander polynomial is at least 4 (see below). To do so we found the need to extend the technology of [COT1] to a "relative" setting, and to greatly generalize the key technical result of Cochran and Teichner [CT, Theorem 4.3]. We expect that this extended technology will be of independent interest, enabling further work in this area.

In this paper, we introduce the notion of an n-cylinder (Definition 2.1), generalizing the notion of an $n$-solution of COT . The difference is that an $n$-solution allows only one boundary component, whereas an $n$-cylinder can have multiple boundary components. A basic (trivial) example of an $n$-cylinder (with two boundary components) is a (spin) homology cobordism between the zero surgeries on two knots. If two knots are concordant, then one can easily find such an $n$-cylinder (which in this case is a homology cobordism), by doing surgery on $S^{3} \times[0,1]$ along the annulus cobounded by the knots (Remark 2.2(4)). Using this, we define a family of new equivalence relations on the knot concordance group, that we call $n$-solvequivalence (see Definition 2.5) : $K_{0}$ is $n$-solvequivalent to $K_{1}$ if the zero-framed surgery on $K_{0}$ and the zero-framed surgery on $K_{1}$ cobound an $n$-cylinder. From the basic example above, it is clear that concordant knots are $n$-solvequivalent for all $n$.

On the other hand, the filtration $\mathcal{F}$ of COT immediately suggests another family of equivalence relations on $\mathcal{C}$, given as follows. We say $K_{0}$ and $K_{1}$ are concordant modulo $n$-solvability if $K_{0} \#\left(-K_{1}\right)$ is $n$-solvable. There is a close relationship between $n$-solvability and $n$-solvequivalence. It is not difficult to show that if two knots are concordant modulo $n$-solvability, then they are $n$-solvequivalent (Proposition 2.7), but we have not been able to establish the converse. Hence $n$ solvequivalence is a possibly weaker obstruction to concordance than $n$-solvability. Note that $n$-solvequivalence reflects information on the zero surgeries on each $K_{i}$ separately, but concordance modulo n-solvability reflects only information on the zero surgery on $K_{0} \#\left(-K_{1}\right)$ (see Proposition 3.6).

As an application of $n$-solvequivalence and of our other extensions of the technology of [COT1], [COT2], and [CT], we obtain the following theorem, generalizing the nontriviality results cited above. The condition that the degree of the Alexander polynomial be at least 4 may seem ad hoc. However, very recent work of S. Friedl and Teichner [FT] complements our theorem and we use it to show that our theorem is "best possible", in the sense that it is false for certain knots whose Alexander polynomial has degree 2 .

The reader is referred to [CT, Section 3] for the definition of symmetric Gropes. Let $G$ be a group. Then the $n$-th derived group of $G$ is inductively defined by $G^{(0)} \equiv G$ and $G^{(n+1)} \equiv\left[G^{(n)}, G^{(n)}\right]$.

Theorem 5.1 (Main Theorem). Let $n$ be a positive integer. Let $K$ be a knot whose Alexander polynomial has degree greater than 2 (if $n=1$, then degree equal to 2 is allowed). Then there is an infinite family of knots $\left\{K_{i} \mid i=0,1,2, \ldots\right\}$ with $K_{0}=K$ that satisfies the following:

(1) For each $i, K_{i}-K$ is $n$-solvable. In particular, $K_{i}$ is $n$-solvequivalent to $K$. Moreover, $K_{i}$ and $K$ cobound, in $S^{3} \times[0,1]$, a smoothly embedded symmetric Grope of height $n+2$. 
(2) If $i \neq j$, then $K_{i}$ is not (n.5)-solvequivalent to $K_{j}$. In particular, $K_{i}-K_{j}$ is not (n.5)-solvable, and $K_{i}$ and $K_{j}$ do not cobound, in $S^{3} \times[0,1]$, an embedded symmetric Grope of height $(n+2.5)$.

(3) For each $i, K_{i}$ has the same order $m$ integral higher-order Alexander module as $K$ for $m=0,1, \ldots, n-1$. Indeed, if $G_{i}$ and $G$ denote the knot groups of $K_{i}$ and $K$, respectively, then there is an isomorphism $G_{i} /\left(G_{i}\right)^{(n+1)} \rightarrow$ $G / G^{(n+1)}$ that preserves the peripheral structures.

(4) For each $i, K_{i}$ has the same $m^{\text {th }}$-higher-order Seifert presentation as $K$ for $m=0,1, \ldots, n-1$. In particular, all of the knots admit the same classical Seifert matrix.

(5) If $i>j, K_{i}-K_{j}$ is of infinite order in $\mathcal{F}_{(n)} / \mathcal{F}_{(n .5)}$.

(6) If $i, j>0, s\left(K_{i}\right)=s\left(K_{j}\right)$ and $\tau\left(K_{i}\right)=\tau\left(K_{j}\right)$, where $s$ is Rasmussen's smooth concordance invariant and $\tau$ is the smooth concordance invariant of P. Ozsváth and Z. Szabó ([Ra], [OS]).

The general term higher-order Alexander modules was first used in [COT1]. The higher-order Alexander modules and higher-order Seifert presentations of a knot that we use here were introduced and developed by Cochran [C]. These are defined in terms of the homology of the derived covers of the knot exterior. Definitions are given in Section 5. But, in particular, these generalize (the case $n=0$ ) the classical Alexander module and Seifert matrix. Therefore, the case $n=1$ of our theorem recovers the previously mentioned results of Livingston and Kim. If the degree of the Alexander polynomial is precisely 2, then the Livingston-Kim result says that it is possible to fix the classical Seifert matrix $(m=0)$, but we show, using recent work of Friedl and Teichner, that there are at least some such knots for which it is not possible to fix any higher-order Seifert presentation matrices. Specifically, the main theorem fails for $n>1$ if we allow knots whose Alexander polynomials have degree 2 (see Proposition 5.10). More details are within.

As another obvious corollary we recover the aforementioned result of Cochran and Teichner [CT].

Corollary 5.2. For every positive integer $n, \mathcal{F}_{(n)} / \mathcal{F}_{(n .5)}$ has positive rank.

Indeed, this corollary is essentially equivalent to parts (1) and (5) in Theorem 5.1, and so our new contribution lies in being able to impose (3) and (4).

Now consider the filtration of $\mathcal{C}$ given by the subgroups $\mathcal{G}_{n}$ of knots that bound topologically embedded symmetric Gropes of height $n$ in $B^{4}$ and the filtration of the smooth knot concordance group $\mathcal{C}^{\text {smooth }}$ given by the subgroups $\mathcal{G}_{n}^{\text {smooth }}$ of knots that bound smoothly embedded symmetric Gropes of height $n$ in $B^{4}$. It was shown in [CT, Theorem 1.4] that $\mathcal{G}_{n+2} / \mathcal{G}_{n+2.5}$ has positive rank if $n \geq 0$. It follows from their work (although not explicitly stated) that $\mathcal{G}_{n+2}^{\text {smooth }} / \mathcal{G}_{n+2.5}^{\text {smooth }}$ has positive rank. Our work recovers these results, together with the added control of the Alexander and Seifert data, and allows for the following additional observations concerning the smooth concordance invariants of Rasmussen and Ozsváth-Szabó.

Corollary 5.3. Let $n$ be a positive integer.

1. $\mathcal{G}_{n+2}^{\text {smooth }} / \mathcal{G}_{n+2.5}^{\text {smooth }}$ has elements of infinite order represented by knots $K$ with $s(K)=0$ and $\tau(K)=0$.

2. The kernel of the homomorphism $S T: \mathcal{G}_{n+2}^{\text {smooth }} \rightarrow \mathbb{Z} \times \mathbb{Z}$ given by $S T(K)=$ $(s(K), \tau(K))$ is infinite. 
Our Proposition 6.3 and Theorem 6.4 represent a significant strengthening of the crucial technical results of Proposition 6.2 and Theorem 6.3 in [CT]. In the process, we introduce the notion of an algebraic $n$-solution which should be considered as an algebraic abstraction of our notion of an $n$-cylinder (Definition 6.1 and Proposition 6.3). In fact, an algebraic $n$-solution was introduced by Cochran and Teichner in $[\mathrm{CT}]$, but our notion is much more general.

For an obstruction to $n$-solvequivalence, as in [COT1], [COT2], and [CT], we use the Cheeger-Gromov von Neumann $\rho$-invariant (Theorem 4.2 and Corollary 4.2 in $[\mathrm{ChG}])$. For this purpose, we analyze the twisted homology groups of an $n$-cylinder and its boundary in Section 3.

This paper is organized as follows. In Section 2, we define an $n$-cylinder and $n$-solvequivalence. We also investigate the relationship between $n$-solvability and $n$-solvequivalence. In Section 3, the twisted homology groups of an $n$-cylinder and its boundary are analyzed. In Section 4, we explain how to realize von Neumann $\rho$-invariants as obstructions to $n$-solvequivalence. All of these sections generalize [COT1] by extending to "the relative case". The main theorem is proved in Section 5 . In the last section, we define and investigate an algebraic $n$-solution. Here, we not only generalize $[\mathrm{CT}]$ to the relative case but also significantly strengthen a primary technical tool. In a strictly logical order, Section 6 would precede Section 5 , but we have chosen to place Section 6 after Section 5, since the arguments in Section 6 are very technical.

\section{2. $n$-Cylinders AND $n$-SOLVEQUivalenCE}

In this section, we define an $n$-cylinder and $n$-solvequivalence. Throughout this paper integer coefficients are understood for homology groups unless specified otherwise.

Recall that a group $G$ is called $n$-solvable if $G^{(n+1)}=1$. If $X$ is a topological space, $X^{(n)}$ denotes the covering space of $X$ corresponding to the $n$-th derived group of $\pi_{1}(X)$. For a compact spin 4-manifold $W$ there is an equivariant intersection form

$$
\lambda_{n}: H_{2}\left(W^{(n)}\right) \times H_{2}\left(W^{(n)}\right) \longrightarrow \mathbb{Z}\left[\pi_{1}(W) / \pi_{1}(W)^{(n)}\right]
$$

and a self-intersection form $\mu_{n}$ ([Wa, Chapter 5], [COT1, Section 7]). (Here $H_{2}\left(W^{(n)}\right)$ is considered to be a right $\mathbb{Z}\left[\pi_{1}(W) / \pi_{1}(W)^{(n)}\right]$-module.) In general, these intersection forms are singular.

Let us denote the boundary components of $W$ by $M_{i}(i=1,2, \ldots, \ell)$. That is, $\partial W=\coprod_{i=1}^{\ell} M_{i}$ where each $M_{i}$ is a connected 3 -manifold. Let $I \equiv$ Image inc $_{*}$ : $\left.H_{2}(\partial W) \rightarrow H_{2}(W)\right\}$ where inc $_{*}$ is the homomorphism induced by the inclusion from $\partial W$ to $W$. Then the usual intersection form factors through

$$
\overline{\lambda_{0}}: H_{2}(W) / I \times H_{2}(W) / I \longrightarrow \mathbb{Z} .
$$

We define an $n$-Lagrangian to be a $\mathbb{Z}\left[\pi_{1}(W) / \pi_{1}(W)^{(n)}\right]$-submodule of $H_{2}\left(W^{(n)}\right)$ on which $\lambda_{n}$ and $\mu_{n}$ vanish and which maps onto a $\frac{1}{2}$-rank direct summand of $H_{2}(W) / I$ under the covering map. An $n$-surface is defined to be a based and immersed surface in $W$ that can be lifted to $W^{(n)}$. Observe that any class in $H_{2}\left(W^{(n)}\right)$ can be represented by an $n$-surface and that $\lambda_{n}$ can be calculated by counting intersection points in $W$ among representative $n$-surfaces weighted appropriately by signs and by elements of $\pi_{1}(W) / \pi_{1}(W)^{(n)}$. We say an $n$-Lagrangian $L$ admits $m$-duals (for $m \leq n$ ) if $L$ is generated by (lifts of) $n$-surfaces $\ell_{1}, \ell_{2}, \ldots, \ell_{g}$ 
and there exist $m$-surfaces $d_{1}, d_{2}, \ldots, d_{g}$ such that $H_{2}(W) / I$ has rank $2 g$ and $\lambda_{m}\left(\ell_{i}, d_{j}\right)=\delta_{i, j}$. Similarly we can define a rational $n$-Lagrangian and rational $m$-duals. Here we do not require that $W$ be spin and $\mu_{n}$ is not discussed. A rational $n$-Lagrangian $L$ is a $\mathbb{Q}\left[\pi_{1}(W) / \pi_{1}(W)^{(n)}\right]$-submodule of $H_{2}\left(W^{(n)} ; \mathbb{Q}\right)$ on which $\lambda_{n}$ (with $\mathbb{Q}\left[\pi_{1}(W) / \pi_{1}(W)^{(n)}\right]$ coefficients) vanishes and which maps onto a $\frac{1}{2}$-rank direct summand of $H_{2}(W ; \mathbb{Q}) / I_{\mathbb{Q}}$ under the covering map. Here $I_{\mathbb{Q}} \equiv$ Image $_{\text {inc }}$ : $\left.H_{2}(\partial W ; \mathbb{Q}) \rightarrow H_{2}(W ; \mathbb{Q})\right\}$. We say a rational $n$-Lagrangian $L$ admits rational $m$-duals (for $m \leq n$ ) if $L$ is generated by $n$-surfaces $\ell_{1}, \ell_{2}, \ldots, \ell_{g}$ and there are $m$-surfaces $d_{1}, d_{2}, \ldots, d_{g}$ such that $H_{2}(W ; \mathbb{Q}) / I_{\mathbb{Q}}$ has $\operatorname{rank} 2 g$ and $\lambda_{m}\left(\ell_{i}, d_{j}\right)=\delta_{i, j}$.

Definition 2.1. Let $n$ be a nonnegative integer.

(1) A compact, connected, spin 4-manifold $W$ with $\partial W=\coprod_{i=1}^{\ell} M_{i}$ where each $M_{i}$ is a connected component of $\partial W$ with $H_{1}\left(M_{i}\right) \cong \mathbb{Z}$ is an $n$-cylinder if the inclusion from $M_{i}$ to $W$ induces an isomorphism on $H_{1}\left(M_{i}\right)$ for each $i$ and $W$ admits an $n$-Lagrangian with $n$-duals. In addition to this, if the $n$-Lagrangian is the image of an $(n+1)$-Lagrangian under the covering map, then $W$ is called an (n.5)-cylinder.

(2) A compact, connected 4-manifold $W$ with $H_{1}(W ; \mathbb{Q}) \cong \mathbb{Q}$ and with $\partial W=$ $\coprod_{i=1}^{\ell} M_{i}$ where each $M_{i}$ is a connected component of $\partial W$ with $H_{1}\left(M_{i}\right) \cong \mathbb{Q}$ is a rational $n$-cylinder of multiplicity $\left\{m_{1}, m_{2}, \ldots, m_{\ell}\right\}\left(m_{i} \in \mathbb{Z}\right)$ if the inclusion from $M_{i}$ to $W$ induces an isomorphism on $H_{1}\left(M_{i} ; \mathbb{Q}\right)$ such that a generator 1 in $H_{1}\left(M_{i}\right)$ /torsion is sent to $m_{i}$ in $H_{1}(W) /$ torsion and $W$ admits a rational $n$-Lagrangian with rational $n$-duals. In addition to this, if the rational $n$-Lagrangian is the image of a rational $(n+1)$-Lagrangian under the covering map, then $W$ is called a rational (n.5)-cylinder of multiplicity $\left\{m_{1}, m_{2}, \ldots, m_{\ell}\right\}$.

Remark 2.2. (1) By naturality of intersection forms and commutativity of the diagram below, the image of an $n$-Lagrangian in $H_{2}(W) / I$ becomes a metabolizer for the $\overline{\lambda_{0}}$, and it follows that the signature of $W$ is zero. The same is true for a rational $n$-Lagrangian.

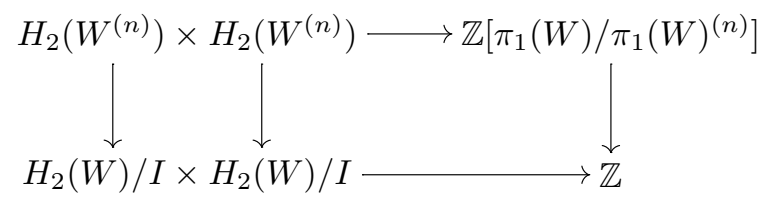

(2) Using twisted local coefficients, $H_{2}\left(W^{(n)}\right)$ is identified with $H_{2}(W$; $\left.\mathbb{Z}\left[\pi_{1}(W) / \pi_{1}(W)^{(n)}\right]\right)$ as (right) $\mathbb{Z}\left[\pi_{1}(W) / \pi_{1}(W)^{(n)}\right]$-modules.

(3) An $n$-cylinder is, in particular, a rational $n$-cylinder of multiplicity $\{1,1$, $\ldots, 1\}$.

(4) A spin homology cobordism, say $W$, between two closed, connected, oriented 3-manifolds is an $n$-cylinder since $H_{2}(W) / I=0$.

The notion of an $n$-cylinder extends the notion of an $n$-solution in [COT1, Definitions $1.2,8.5,8.7]$. This is clear from the following proposition.

Proposition 2.3. A 4-manifold $W$ with one boundary component is an $n$-cylinder if and only if it is an n-solution. 
Proof. Suppose $V$ is a compact, connected, spin 4-manifold with $\partial V=N$ where $N$ is connected, $H_{1}(N) \cong \mathbb{Z}$, and the inclusion from $N$ to $V$ induces an isomorphism

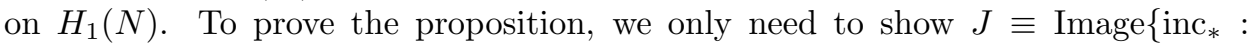
$\left.H_{2}(N) \longrightarrow H_{2}(V)\right\}=0$ where inc $_{*}$ is the homomorphism induced by the inclusion. Since the inclusion induces an isomorphism on the first homology, inc ${ }^{*}: H^{1}(V) \longrightarrow$ $H^{1}(N)$ is an isomorphism. By Poincaré duality, the boundary map $H_{3}(V, N) \longrightarrow$ $H_{2}(N)$ is an isomorphism. Hence inc in $_{2} H_{2}(N) \longrightarrow H_{2}(V)$ is a zero homomorphism in the homology long exact sequence of the pair $(V, N)$.

Remark 2.4. In a similar fashion, one can easily see that a 4 -manifold $W$ with one boundary component is a rational $n$-cylinder if and only if it is a rational $n$-solution (see [COT1, Definition 4.1]).

Using $n$-cylinders, we define $n$-solvequivalence between 3 -manifolds and between knots.

Definition 2.5. Let $n \in \frac{1}{2} \mathbb{N}_{0}$. Let $M_{1}$ and $M_{2}$ be closed, connected, oriented 3-manifolds.

(1) If there exists an $n$-cylinder $W$ such that $\partial W=M_{1} \amalg-M_{2}$, then $M_{1}$ is $n$-solvequivalent to $M_{2}$ via $W$.

(2) If there exists a rational $n$-cylinder (of multiplicity $\left\{m_{1},-m_{2}\right\}$ ) $W$ such that $\partial W=M_{1} \amalg-M_{2}$, then $M_{1}$ is rationally $n$-solvequivalent to $M_{2}$ via $W$ (of multiplicity $\left\{m_{1}, m_{2}\right\}$ ).

(3) For two given knots $K_{i}(i=1,2)$, if the zero framed surgery on $S^{3}$ along $K_{1}$ is (rationally) $n$-solvequivalent to the zero framed surgery on $S^{3}$ along $K_{2}$ via $W$, then we say $K_{1}$ is (rationally) $n$-solvequivalent to $K_{2}$ via $W$.

It is not hard to show that $n$-solvequivalence is an equivalence relation. Here we give a proof only for transitivity.

Proposition 2.6. Let $L, M$, and $N$ be closed, connected, oriented 3-manifolds with $H_{1}(L) \cong H_{1}(M) \cong H_{1}(N) \cong \mathbb{Z}$. Let $n \in \frac{1}{2} \mathbb{N}_{0}$. Suppose $L$ is $n$-solvequivalent to $M$ via $V$ and $M$ is n-solvequivalent to $N$ via $W$. Then $L$ is $n$-solvequivalent to $N$ via $V \cup_{M} W$.

Proof. Let $n \in \mathbb{N}$. Let $X \equiv V \cup_{M} W$. Then $\partial X=L \amalg-N$. We need to show that $X$ is an $n$-cylinder. The proof for the condition on the first homology groups is not hard, hence left for the readers. Denote the inclusion map from $A$ to $B$ by $i_{A, B}$ for two topological spaces $A \subset B$. Using the long exact sequences of pairs, one can prove that $H_{2}(V) /\left(i_{\partial V, V}\right)_{*}\left(H_{2}(\partial V)\right) \cong H_{2}(V) /\left(i_{L, V}\right)_{*}\left(H_{2}(L)\right) \cong$ $H_{2}(V) /\left(i_{M, V}\right)_{*}\left(H_{2}(M)\right)$. We have similar isomorphisms for $(W, M \amalg-N)$. Now from the Mayer-Vietoris sequence for $V$ and $W$ along $M$, one can see that

$$
\begin{aligned}
\left(H_{2}(V) /\left(i_{\partial V, V}\right)_{*}\left(H_{2}(\partial V)\right)\right) & \oplus\left(H_{2}(W) /\left(i_{\partial W, W}\right)_{*}\left(H_{2}(\partial W)\right)\right) \\
& \cong H_{2}(X) /\left(i_{\partial X, X}\right)_{*}\left(H_{2}(\partial X)\right) .
\end{aligned}
$$

Since $\left(i_{V, X}\right)_{*}$ and $\left(i_{W, X}\right)_{*}$ map $\pi_{1}(V)^{(n)}$ and $\pi_{1}(W)^{(n)}$ into $\pi_{1}(X)^{(n)}$, respectively, and intersection forms are natural, the "union" of the $n$-Lagrangian with $n$-duals for $V$ and the $n$-Lagrangian with $n$-duals for $W$ constitutes an $n$-Lagrangian with $n$-duals for $X$.

The proof for the case when $n$ is a half-integer is left to the reader. 
The notion of $n$-solvability defined by COT suggests an equivalence relation on $\mathcal{C}$ wherein $K_{0} \sim K_{1}$ if and only if $K_{0} \#\left(-K_{1}\right)$ is $n$-solvable. This relation has not been given a name. The following proposition reveals the close connection, for knots, between $n$-solvequivalence and the equivalence relation arising from COT's $n$-solvability. We initially expected that these two equivalence relations were in fact identical. However, we have not proved this and now believe that they may be slightly different.

Proposition 2.7. Let $n \in \frac{1}{2} \mathbb{N}_{0}$. For knots $K_{0}$ and $K_{1}$, if $K_{0} \#\left(-K_{1}\right)$ is n-solvable, then $K_{0}$ is n-solvequivalent to $K_{1}$.

Proof. Suppose $n \in \mathbb{N}_{0}$. Let $M_{0}, M_{1}$, and $M$ denote the zero surgeries on $S^{3}$ along $K_{0}, K_{1}$, and $K_{0} \#\left(-K_{1}\right)$, respectively. We construct a (standard) cobordism between $M_{0} \amalg\left(-M_{1}\right)$ and $M$. Take a product $\left(M_{0} \amalg-M_{1}\right) \times[0,1]$. By attaching a 1-handle along $\left(M_{0} \amalg-M_{1}\right) \times\{1\}$ we get a 4 -manifold whose upper boundary is $M_{0} \#\left(-M_{1}\right)$. Note that $M_{0} \#\left(-M_{1}\right)$ is also obtained by taking zero framed surgery on $S^{3}$ along a split link $K_{0} \amalg-K_{1}$. Next add a zero framed 2-handle along the upper boundary of this 4-manifold such that the attaching map is an unknotted circle which links $K_{0}$ and $-K_{1}$ once. Then the resulting 4-manifold has upper boundary $M$ as may be seen by sliding the 2-handle represented by zero surgery on $K_{0}$ over that of $K_{1}$. This 4-manifold is the cobordism $C$ between $M_{0} \amalg-M_{1}$ and $M$. Since $K_{0} \#\left(-K_{1}\right)$ is $n$-solvable, $M$ bounds an $n$-solution $V$. Let $W \equiv C \cup_{M} V$. We claim that $W$ is an $n$-cylinder with $\partial W=M_{0} \amalg-M_{1}$. This will complete the proof.

Note that $\partial W=M_{0} \amalg-M_{1}$. One sees that $H_{2}\left(M_{0}\right)$ and $H_{2}\left(M_{1}\right)$ are generated by capped-off Seifert surfaces for $K_{0}$ and $K_{1}$, respectively. It is easy to show that $H_{2}(C)=H_{2}\left(M_{0}\right) \oplus H_{2}\left(M_{1}\right)(\cong \mathbb{Z} \oplus \mathbb{Z})$ and $H_{1}(C) \cong H_{1}\left(M_{1}\right) \cong H_{1}\left(M_{0}\right) \cong H_{1}(M)$ $(\cong \mathbb{Z})$ using Mayer-Vietoris sequences. Also one sees that the inclusion from $M$ to $C$ induces an injection inc ${ }_{*}: H_{2}(M) \longrightarrow H_{2}(C)$ where $H_{2}(M) \cong \mathbb{Z}$ and inc sends $1(\in \mathbb{Z})$ to $(1,1)(\in \mathbb{Z} \oplus \mathbb{Z})$. Consider the following Mayer-Vietoris sequence:

$$
\cdots \rightarrow H_{2}(M) \stackrel{f}{\rightarrow} H_{2}(C) \oplus H_{2}(V) \rightarrow H_{2}(W) \stackrel{g}{\rightarrow} H_{1}(M) \stackrel{h}{\rightarrow} H_{1}(C) \oplus H_{1}(V) \rightarrow \cdots
$$

Since $h$ is an injection, $g$ is a zero map. Since $H_{3}(V, M) \rightarrow H_{2}(M)$ is a dual map of $H^{1}(V) \rightarrow H^{1}(M)$ which is an isomorphism, by the long exact sequence of homology groups of the pair $(V, M)$, the inclusion induced homomorphism $H_{2}(M) \rightarrow H_{2}(V)$ is a zero map. Thus the image of $f$ in $H_{2}(V)$ is zero and by our previous observation the image of $f$ in $H_{2}(C)$ is isomorphic to $\mathbb{Z}$ generated by $(1,1)$ in $H_{2}(C)$. Therefore $H_{2}(W) \cong H_{2}(V) \oplus \mathbb{Z}$. Furthermore the last $\mathbb{Z}$ summand on the righthand side is exactly Image $\left\{\right.$ inc $\left._{*}: H_{2}(\partial W) \longrightarrow H_{2}(W)\right\}$, which is denoted by $I$. Hence $H_{2}(W) / I \cong H_{2}(V)$. Since $V$ is a subspace of $W, \pi_{1}(V)^{(n)}$ is a subgroup of $\pi_{1}(W)^{(n)}$. This implies that $k$-surfaces in $V$ are also $k$-surfaces in $W$ for every integer $k$. Now by naturality of (equivariant) intersections forms, one can prove that an $n$-Lagrangian with $n$-duals for $V$ maps to an $n$-Lagrangian with $n$-duals for $W$.

Clearly $W$ is a compact, connected 4-manifold, and it only remains to show $W$ is spin. But this is obvious since $C$ and $V$ are spin, and when we take the union of $C$ and $V$ we can adjust the spin structure of either $C$ or $V$ to make $W$ spin. 
Finally, in the case that $K_{0} \#\left(-K_{1}\right)$ is $(n .5)$-solvable via $V, K_{0}$ is $(n .5)$-solvequivalent to $K_{1}$ via $W$ where $W$ is constructed as above. The argument for a proof for this goes the same as above, and one just needs to notice that an $(n+1)$-Lagrangian with $n$-duals for $V$ maps to an $(n+1)$-Lagrangian with $n$-duals for $W$.

Remark 2.8. There are a couple of natural arguments for attempting to prove the converse of Proposition 2.7, but they do not work completely since capped-off Seifert surfaces for knots do not lift to higher covers of 4-manifolds in the arguments.

\section{HOMOLOGY GROUPS OF AN $n$-CYLINDER AND ITS BOUNDARY}

Let $\Gamma$ be a poly-(torsion-free-abelian) group (abbreviated PTFA). Then $\mathbb{Z} \Gamma$ is an Ore Domain and thus embeds in its classical right ring of quotients $\mathcal{K}_{\Gamma}$, which is a skew field [COT1, Proposition 2.5]. The skew field $\mathcal{K}_{\Gamma}$ is a $\mathbb{Z} \Gamma$-bimodule and has useful properties. In particular, $\mathcal{K}_{\Gamma}$ is flat as a left $\mathbb{Z} \Gamma$-module (see [Ste, Proposition II.3.5]), and every module over $\mathcal{K}_{\Gamma}$ is a free module with a well defined rank over $\mathcal{K}_{\Gamma}$. The rank of any $\mathbb{Z} \Gamma$-module $M$ is then defined to be the $\mathcal{K}_{\Gamma}$-rank of $M \otimes_{\mathbb{Z} \Gamma} \mathcal{K}_{\Gamma}$. Now we investigate $H_{0}, H_{1}$, and $H_{2}$ of an $n$-cylinder $W$ and its boundary with coefficients in $\mathbb{Z} \Gamma$ or $\mathcal{K}_{\Gamma}$. Throughout this section, $\Gamma$ denotes a PTFA group and $\mathcal{K}_{\Gamma}$ its (skew) quotient field of fractions. The following two basic propositions are due to [COT1].

Proposition 3.1 ([COT1, Proposition 2.9]). Suppose $X$ is a $C W$-complex and there is a homomorphism $\phi: \pi_{1}(X) \longrightarrow \Gamma$. Suppose $\psi: \mathbb{Z} \Gamma \rightarrow \mathcal{R}$ defines $\mathcal{R}$ as a $\mathbb{Z} \Gamma$-bimodule and some element of the augmentation ideal of $\mathbb{Z}\left[\pi_{1}(X)\right]$ is invertible in $\mathcal{R}$. Then $H_{0}(X ; \mathcal{R})=0$. In particular, if $\phi: \pi_{1}(X) \rightarrow \Gamma$ is a nontrivial coefficient system, then $H_{0}\left(X ; \mathcal{K}_{\Gamma}\right)=0$.

Proposition 3.2 ([COT1, Proposition 2.11]). Suppose $X$ is a $C W$-complex such that $\pi_{1}(X)$ is finitely generated, and $\phi: \pi_{1}(X) \longrightarrow \Gamma$ is a nontrivial coefficient system. Then

$$
\operatorname{rk}_{\mathcal{K}_{\Gamma}} H_{1}\left(X ; \mathcal{K}_{\Gamma}\right) \leq \beta_{1}(X)-1
$$

In particular, if $\beta_{1}(X)=1$, then $H_{1}\left(X ; \mathcal{K}_{\Gamma}\right)=0$. That is, $H_{1}(X ; \mathbb{Z} \Gamma)$ is a $\mathbb{Z} \Gamma$ torsion module.

Since a rational $n$-cylinder $W$ has $\beta_{1}(W)=1$ we have:

Corollary 3.3. Suppose $W$ is a rational n-cylinder with $\partial W=\coprod_{i=1}^{\ell} M_{i}$ where each $M_{i}$ is a connected component of $\partial W$. If $\phi: \pi_{1}(W) \longrightarrow \Gamma$ is a nontrivial coefficient system, then $H_{0}\left(W ; \mathcal{K}_{\Gamma}\right)=H_{1}\left(W ; \mathcal{K}_{\Gamma}\right)=0$ and $H_{0}\left(M_{i} ; \mathcal{K}_{\Gamma}\right)=H_{1}\left(M_{i} ; \mathcal{K}_{\Gamma}\right)=0$ for all $i$. Moreover, $H_{2}\left(M_{i} ; \mathcal{K}_{\Gamma}\right)=0$ for all $i$.

Proof. A short technical argument shows that the restriction of the coefficient system to each $\pi_{1}\left(M_{i}\right)$ is nontrivial (see the proof of [COT1, Proposition 2.11]). The proof for $H_{0}$ and $H_{1}$ follows from the previous two propositions and the definition of a rational $n$-cylinder. Notice that $H_{2}\left(M_{i} ; \mathcal{K}_{\Gamma}\right) \cong H^{1}\left(M_{i} ; \mathcal{K}_{\Gamma}\right) \cong H_{1}\left(M_{i} ; \mathcal{K}_{\Gamma}\right)=0$ by Poincaré duality and the universal coefficient theorem.

The following proposition about $H_{2}$ plays an essential role in showing that von Neumann $\rho$-invariants obstruct $n$-solvequivalence. It extends Proposition 4.3 of 
[COT1]. For the case that $W$ has one boundary component, refer to Proposition 4.3 in $[\mathrm{COT} 1]$. Let $I_{\mathbb{Q}} \equiv \operatorname{Image}\left\{\mathrm{inc}_{*}: H_{2}(\partial W ; \mathbb{Q}) \rightarrow H_{2}(W ; \mathbb{Q})\right\}$.

Proposition 3.4. Suppose $W$ is a compact, connected, oriented 4-manifold with $\partial W=M_{1} \amalg M$ where $M=\coprod_{i=2}^{\ell} M_{i}$ and $M_{i}$ are connected, $i=1,2, \ldots, \ell(\ell \geq 2)$, and, for all $i, H_{1}\left(M_{i} ; \mathbb{Q}\right) \cong H_{1}(W ; \mathbb{Q}) \cong \mathbb{Q}$, induced by inclusion. Suppose $\phi$ : $\pi_{1}(W) \longrightarrow \Gamma$ is a nontrivial (PTFA) coefficient system. Then

$$
\operatorname{rk}_{\mathcal{K}_{\Gamma}} H_{2}\left(W ; \mathcal{K}_{\Gamma}\right)=\operatorname{rk}_{\mathbb{Q}}\left(H_{2}(W ; \mathbb{Q}) / I_{\mathbb{Q}}\right)=\left(\operatorname{rk}_{\mathbb{Q}} H_{2}(W ; \mathbb{Q})\right)-(\ell-1) .
$$

Moreover, suppose there are 2-dimensional surfaces $S_{j}$ and continuous maps $f_{j}$ : $S_{j} \longrightarrow W(j \in J)$ that are lifted to $\tilde{f}_{j} \longrightarrow W_{\Gamma}$ where $W_{\Gamma}$ is a regular $\Gamma$-cover associated to $\phi$. If $\left\{\left[f_{j}\right] \mid j \in J\right\}$ is linearly independent in $H_{2}(W, M ; \mathbb{Q})$, then $\left\{\left[\tilde{f}_{j}\right] \mid j \in J\right\}$ is $\mathbb{Q} \Gamma$-linearly independent in $H_{2}(W, M ; \mathbb{Q} \Gamma)$.

Proof. Since $W$ has nontrivial boundary, we can choose a 3-dimensional $C W$ complex structure for $(W, \partial W)$. Let $C_{*}(W)$ be a cellular chain complex associated to a chosen $C W$-complex structure with coefficients in $\mathbb{Q}$. Let $C_{*}\left(W_{\Gamma}\right)$ be the corresponding free $\mathcal{K}_{\Gamma}$ chain complex of $W_{\Gamma}$ that is freely generated on cells of $W$. Let $b_{i} \equiv \operatorname{rk}_{\mathcal{K}_{\Gamma}} H_{i}\left(W ; \mathcal{K}_{\Gamma}\right)$ and $\beta_{i} \equiv \operatorname{rk}_{\mathbb{Q}} H_{i}(W ; \mathbb{Q})$ for $1 \leq i \leq 4$. In this proof all chain complexes and homology groups are with coefficients in $\mathbb{Q}$ unless specified otherwise.

By Corollary 3.3, $b_{0}=b_{1}=0$. By Poincaré duality and the universal coefficient theorem, $H_{3}\left(W ; \mathcal{K}_{\Gamma}\right) \cong H^{1}\left(W, \partial W ; \mathcal{K}_{\Gamma}\right) \cong H_{1}\left(W, \partial W ; \mathcal{K}_{\Gamma}\right)$. Using the long exact sequence for the pair $(W, \partial W)$ with coefficients in $\mathcal{K}_{\Gamma}$, Proposition 3.1 and Proposition 3.2 , one sees that $H_{1}\left(W, \partial W ; \mathcal{K}_{\Gamma}\right)=0$. Here we also need that the coefficient system restricted to each $M_{i}$ is nontrivial as mentioned previously (see proof of [COT1, Proposition 2.11]). Hence $b_{3}=0$.

On the other hand, $H_{3}(W) \cong H^{1}(W, \partial W) \cong H_{1}(W, \partial W)$. In the following long exact sequence

$$
\begin{aligned}
\cdots \rightarrow H_{1}(\partial W) \stackrel{f}{\rightarrow} H_{1}(W) & \rightarrow H_{1}(W, \partial W) \rightarrow H_{0}(\partial W) \\
& \stackrel{g}{\rightarrow} H_{0}(W) \rightarrow H_{0}(W, \partial W) \rightarrow 0,
\end{aligned}
$$

the maps $f$ and $g$ are surjective. Therefore $H_{1}(W, \partial W) \cong \mathbb{Q}^{\ell-1}$ and $\beta_{3}=\ell-1$. It is clear that $\beta_{0}=\beta_{1}=1$. Since the Euler characteristics of $C_{*}(W)$ and $C_{*}\left(W_{\Gamma}\right)$ are equal, we deduce that $b_{2}=\beta_{2}-(\ell-1)$.

For $\operatorname{rk}_{\mathbb{Q}}\left(H_{2}(W) / I_{\mathbb{Q}}\right)$, use the following long exact sequence:

$$
H_{2}(\partial W) \rightarrow H_{2}(W) \rightarrow H_{2}(W, \partial W) \rightarrow H_{1}(\partial W) \stackrel{f}{\rightarrow} H_{1}(W) .
$$

Since $f$ is a surjection from $\mathbb{Q}^{\ell}$ to $\mathbb{Q}$, we have an exact sequence as follows:

$$
0 \rightarrow H_{2}(W) / I_{\mathbb{Q}} \rightarrow H_{2}(W, \partial W) \rightarrow \mathbb{Q}^{\ell-1} \rightarrow 0 .
$$

Thus $\operatorname{rk}_{\mathbb{Q}}\left(H_{2}(W) / I_{\mathbb{Q}}\right)=\beta_{2}-(\ell-1)$. This completes the first part of the proof.

For the second part, let $X$ be the one point union of $S_{j}$ using some base paths. Let $f: X \longrightarrow W$ and $\tilde{f}: X \longrightarrow W_{\Gamma}$ be maps which restrict to $f_{j}$ and $\tilde{f}_{j}$, respectively. By taking mapping cylinders, we may think of $X$ as a (2-dimensional) subcomplex of $W$ and $C_{*}(X)$ as a subcomplex of $C_{*}(W)$. If we denote by $X_{\Gamma}$ the regular $\Gamma$-cover associated to $\phi \circ f_{*}$ (which can be thought of as a subcomplex of $\left.W_{\Gamma}\right)$, then $C_{*}\left(X_{\Gamma}\right)$ is a subcomplex of $C_{*}\left(W_{\Gamma}\right)$. In fact, by our hypothesis $f_{j}$ lifts to $\tilde{f}_{j}$, and this implies that $\phi \circ f_{*}$ is trivial on $\pi_{1}(X)$. Hence $X_{\Gamma}$ is a trivial cover which 
consists of $\Gamma$ copies of $X$. Consider the following commutative diagram where each row is exact.

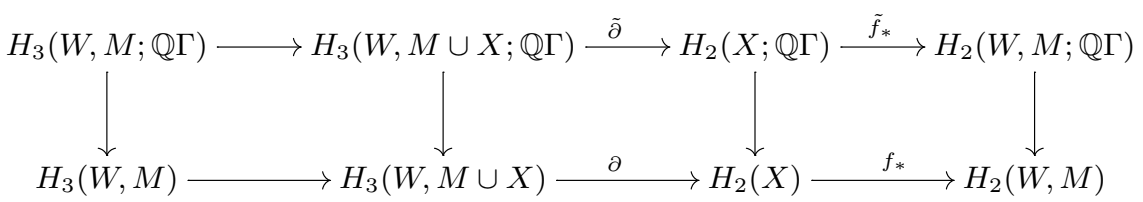

Since $X_{\Gamma}$ is a trivial cover, $H_{2}(X ; \mathbb{Q} \Gamma)$ is a free $\mathbb{Q} \Gamma$-module on $\left\{\left[S_{j}\right]\right\}$. Thus to complete the proof, we need to show $\tilde{f}_{*}$ is injective. Since $H_{3}(W, M) \cong H^{1}\left(W, M_{1}\right) \cong$ $H_{1}\left(W, M_{1}\right)=0$ and $C_{*}(W, M)$ is a 3 -dimensional chain complex, $\partial_{\#}: C_{3}(W, M) \rightarrow$ $C_{2}(W, M)$ is injective. Notice that $C_{*}(W, M)$ can be identified with $C_{*}\left(W_{\Gamma}, M_{\Gamma}\right)$ $\otimes_{\mathbb{Q} \Gamma} \mathbb{Q}$ (similarly for $C_{*}(X)$ and $C_{*}(W, M \cup X)$ ). Then by [Str, pg. 305] (or see [COT1, Proposition 2.4]), $\partial_{\#}: C_{3}\left(W_{\Gamma}, M_{\Gamma}\right) \longrightarrow C_{2}\left(W_{\Gamma}, M_{\Gamma}\right)$ is also injective. Hence we obtain $H_{3}(W, M ; \mathbb{Q} \Gamma)=0$. Now it suffices to show $H_{3}(W, M \cup X ; \mathbb{Q} \Gamma)$ $=0$. Since $f_{*}$ is injective by our hypothesis, $H_{3}(W, M \cup X)=0$. Since $C_{*}(W, M \cup X)$ is a 3-dimensional chain complex, this implies that $\partial_{\#}: C_{3}(W, M \cup X) \longrightarrow$ $C_{2}(W, M \cup X)$ is injective. Once again by [Str, pg. 305], $\partial_{\#}: C_{3}\left(W_{\Gamma}, M_{\Gamma} \cup X_{\Gamma}\right) \longrightarrow$ $C_{2}\left(W_{\Gamma}, M_{\Gamma} \cup X_{\Gamma}\right)$ is injective. Therefore $H_{3}(W, M \cup X ; \mathbb{Q} \Gamma)=0$.

We now investigate the relationship between the first homology groups of an $n$-cylinder and its boundary components. The following lemma generalizes [COT1, Lemma 4.5]. It is the linchpin in proving Theorem 3.8, which establishes the crucial connection between $n$-solvequivalence and homology.

Lemma 3.5. Let $W$ be a rational $n$-cylinder with $M$ as one of its boundary components. Let $\Gamma$ be an $(n-1)$-solvable (PTFA) group and $\mathcal{R}$ be a ring such that $\mathbb{Q} \Gamma \subset \mathcal{R} \subset \mathcal{K}_{\Gamma}$. Suppose $\phi: \pi_{1}(M) \longrightarrow \Gamma$ is a nontrivial coefficient system that extends to $\psi: \pi_{1}(W) \longrightarrow \Gamma$. Then

$$
T H_{2}(W, M ; \mathcal{R}) \stackrel{\partial}{\rightarrow} H_{1}(M ; \mathcal{R}) \stackrel{i_{*}}{\rightarrow} H_{1}(W ; \mathcal{R})
$$

is exact. (Here for an $\mathcal{R}$-module $\mathcal{M}, T \mathcal{M}$ denotes the $\mathcal{R}$-torsion submodule of $\mathcal{M}$.)

Proof. We need to show that every element of $\operatorname{Ker}\left(i_{*}\right)$ is in the image of an element of $T H_{2}(W, M ; \mathcal{R})$. Let $m=\frac{1}{2} \operatorname{rk}_{\mathbb{Q}}\left(H_{2}(W ; \mathbb{Q}) / I_{\mathbb{Q}}\right)$ where $I_{\mathbb{Q}} \equiv$ Image $\left\{\right.$ inc $_{*}$ : $\left.H_{2}(\partial W ; \mathbb{Q}) \rightarrow H_{2}(W ; \mathbb{Q})\right\}$. By Proposition $3.4, \operatorname{rk}_{\mathcal{K}_{\Gamma}} H_{2}(W ; \mathcal{R})=2 m$. Let $\left\{\ell_{1}, \ell_{2}\right.$, $\left.\ldots, \ell_{m}\right\}$ generate a rational $n$-Lagrangian for $W$ and $\left\{d_{1}, d_{2}, \ldots, d_{m}\right\}$ be its $n$-duals. Since $\Gamma$ is $(n-1)$-solvable, $\psi$ descends to $\psi^{\prime}: \pi_{1}(W) / \pi_{1}(W)^{(n)} \longrightarrow \Gamma$. We denote by $\ell_{i}^{\prime}$ and $d_{i}^{\prime}$ the images of $\ell_{i}$ and $d_{i}$ in $H_{2}(W ; \mathcal{R})$. By naturality of intersection forms, the intersection form $\lambda$ defined on $H_{2}(W ; \mathcal{R})$ vanishes on the module generated by $\left\{\ell_{1}^{\prime}, \ell_{2}^{\prime}, \ldots, \ell_{m}^{\prime}\right\}$. Let $\mathcal{R}^{m} \oplus \mathcal{R}^{m}$ be the free module on $\left\{\ell_{i}^{\prime}, d_{i}^{\prime}\right\}$. The following composition

$$
\mathcal{R}^{m} \oplus \mathcal{R}^{m} \stackrel{j_{*}}{\longrightarrow} H_{2}(W ; \mathcal{R}) \stackrel{\lambda}{\longrightarrow} H_{2}(W ; \mathcal{R})^{*} \stackrel{j^{*}}{\longrightarrow}\left(\mathcal{R}^{m} \oplus \mathcal{R}^{m}\right)^{*}
$$

is represented by a block matrix

$$
\left(\begin{array}{ll}
0 & I \\
I & X
\end{array}\right)
$$


This matrix has an inverse which is

$$
\left(\begin{array}{cc}
-X & I \\
I & 0
\end{array}\right)
$$

Thus the composition is an isomorphism. This implies that $j_{*}$ is a monomorphism and $j^{*}$ is a (split) epimorphism. Since $j^{*}$ is a split epimorphism between the free $\mathcal{R}$-modules of the same $\mathcal{K}_{\Gamma}$-rank, and $\mathcal{R}$ is an integral domain, $j^{*}$ is an isomorphism. Hence $\lambda$ is a surjection. Now consider the following diagram where the row is the exact sequence of the pair $(W, M)$ :

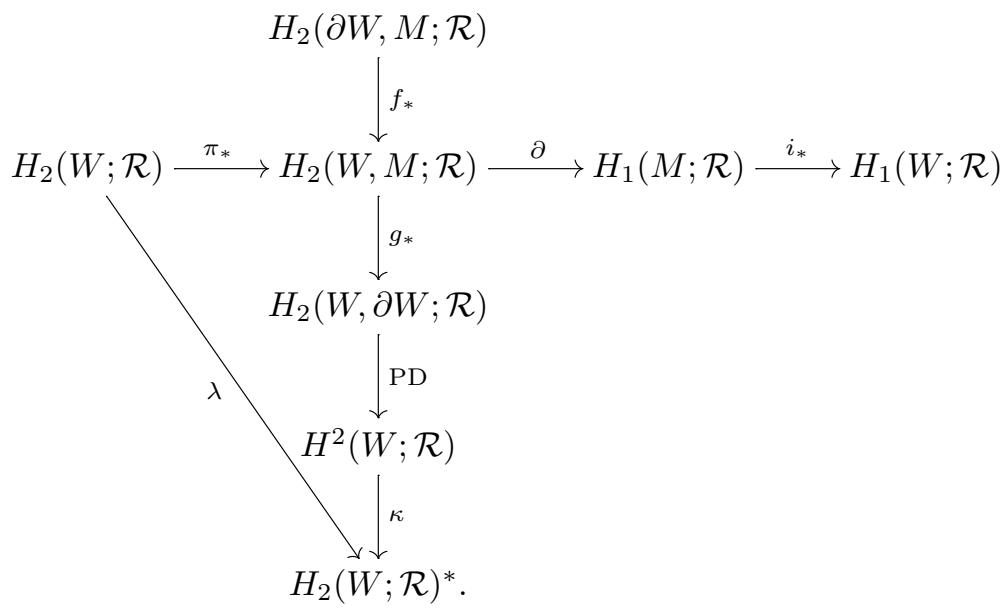

We claim that $\operatorname{Ker}\left(\kappa \circ \mathrm{PD} \circ g_{*}\right)$ is $\mathcal{R}$-torsion. $\operatorname{Ker}(\kappa)$ is $\mathcal{R}$-torsion since it is a split surjection between $\mathcal{R}$-modules of the same rank over $\mathcal{K}_{\Gamma}$. PD is an isomorphism by Poincaré duality. $\operatorname{Ker}\left(g_{*}\right)=\operatorname{Image}\left(f_{*}\right)$ and $H_{2}(\partial W, M ; \mathcal{R})=H_{2}\left(M^{\prime} ; \mathcal{R}\right)$ where $M^{\prime}$ is the disjoint union of the boundary components of $W$ except for $M$. Since $H_{2}\left(M^{\prime} ; \mathcal{R}\right)$ is $\mathcal{R}$-torsion by the flatness of $\mathcal{K}_{\Gamma}$ over $\mathcal{R}$ and Corollary 3.3 (once again we need to know that the restricted coefficient system is nontrivial), $\operatorname{Ker}\left(g_{*}\right)$ is $\mathcal{R}$-torsion. Combining these, one can deduce that $\operatorname{Ker}\left(\kappa \circ \mathrm{PD} \circ g_{*}\right)$ is $\mathcal{R}$-torsion.

Suppose $p \in \operatorname{Ker}\left(i_{*}\right) \subset H_{1}(M ; \mathcal{R})$. Then there exists $x \in H_{2}(W, M ; \mathcal{R})$ such that $\partial(x)=p$. Let $y \in \lambda^{-1}\left(\left(\kappa \circ \mathrm{PD} \circ g_{*}\right)(x)\right)$. Then $x-\pi_{*}(y) \in \operatorname{Ker}\left(\kappa \circ \mathrm{PD} \circ g_{*}\right)$. Hence $x-\pi_{*}(y)$ is $\mathcal{R}$-torsion and $\partial\left(x-\pi_{*}(y)\right)=\partial(x)=p$.

Let $M$ be a closed, connected, oriented 3-manifold with $\beta_{1}(M)=1$. Suppose $\mathcal{R}$ is a PID. Then there exists a nonsingular linking form $\mathcal{B} \ell: H_{1}(M ; \mathcal{R}) \longrightarrow$ $H_{1}(M ; \mathcal{R})^{\#} \equiv \overline{\operatorname{Hom}_{\mathcal{R}}\left(H_{1}(M ; \mathcal{R}), \mathcal{K}_{\Gamma} / \mathcal{R}\right)}$ by [COT1, Theorem 2.13]. This definition and a proof of nonsingularity will be included in our proof of Proposition 3.6. For an $\mathcal{R}$-submodule $P$ of $H_{1}(M ; \mathcal{R})$, we define $P^{\perp} \equiv\left\{x \in H_{1}(M ; \mathcal{R}) \mid \mathcal{B} \ell(x)(y)=\right.$ $0, \forall y \in P\}$, clearly an $\mathcal{R}$-submodule.

Proposition 3.6. Suppose the same hypotheses as in Lemma 3.5. Suppose $\mathcal{R}$ is a PID. Then for $P \equiv \operatorname{Ker}\left\{i_{*}: H_{1}(M ; \mathcal{R}) \longrightarrow H_{1}(W ; \mathcal{R})\right\}, P \subset P^{\perp}$. Moreover, if $M=\partial W$, then $P=P^{\perp}$. 
Proof. Consider the following commutative diagram:

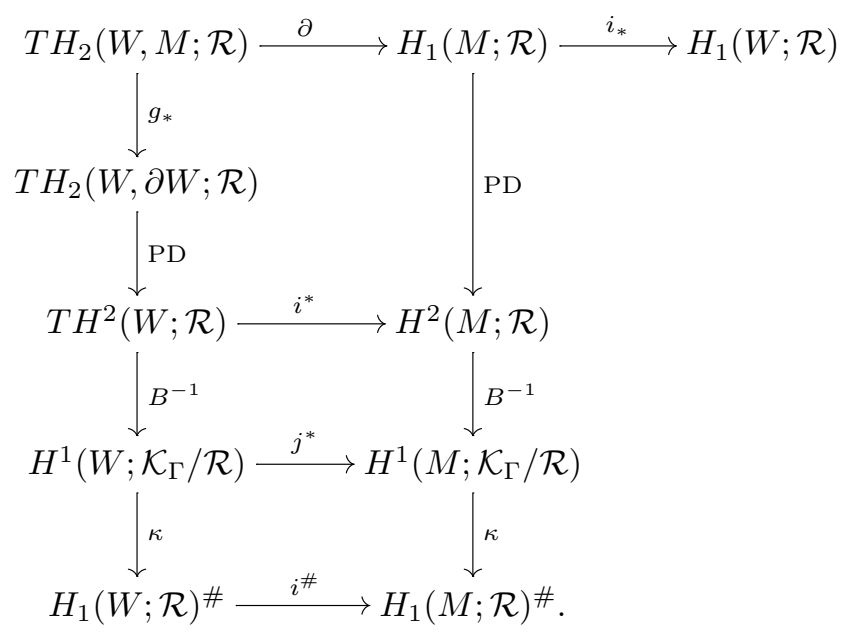

Let $\beta_{\text {rel }}: T H_{2}(W, M ; \mathcal{R}) \longrightarrow H_{1}(W ; \mathcal{R})^{\#}$ be the composition of the maps on the left column and $B \ell: H_{1}(M ; \mathcal{R}) \longrightarrow H_{1}(M ; \mathcal{R})^{\#}$ the composition of the maps on the right column. The PD are induced by Poincaré duality (hence isomorphisms), and $\kappa$ are the Kronecker evaluation maps. $B^{-1}$ are the inverses of the Bockstein homomorphisms. For the existence of $B^{-1}$, we need to show that the Bockstein homomorphisms are isomorphisms. First, we consider the following exact sequence:

$$
H^{1}\left(W ; \mathcal{K}_{\Gamma}\right) \rightarrow H^{1}\left(W ; \mathcal{K}_{\Gamma} / \mathcal{R}\right) \stackrel{B}{\rightarrow} H^{2}(W ; \mathcal{R}) \rightarrow H^{2}\left(W ; \mathcal{K}_{\Gamma}\right) .
$$

Notice that $H^{1}\left(W ; \mathcal{K}_{\Gamma}\right) \cong \operatorname{Hom}_{\mathcal{K}_{\Gamma}}\left(H_{1}\left(W ; \mathcal{K}_{\Gamma}\right), \mathcal{K}_{\Gamma}\right)=0$ by Corollary 3.3 . Since $H^{2}\left(W ; \mathcal{K}_{\Gamma}\right)$ is $\mathcal{R}$-torsion free and $H^{1}\left(W ; \mathcal{K}_{\Gamma} / \mathcal{R}\right)$ is $\mathcal{R}$-torsion, one sees that $B$ is an isomorphism onto $T H^{2}(W ; \mathcal{R})$. Secondly, one can prove that $H^{1}\left(M ; \mathcal{K}_{\Gamma}\right)=$ $H^{2}\left(M ; \mathcal{K}_{\Gamma}\right)=0$ using Corollary 3.3. Hence $B: H^{1}\left(M ; \mathcal{K}_{\Gamma} / \mathcal{R}\right) \longrightarrow H^{2}(M ; \mathcal{R})$ is an isomorphism in the following long exact sequence:

$$
H^{1}\left(M ; \mathcal{K}_{\Gamma}\right) \rightarrow H^{1}\left(M ; \mathcal{K}_{\Gamma} / \mathcal{R}\right) \stackrel{B}{\rightarrow} H^{2}(M ; \mathcal{R}) \rightarrow H^{2}\left(M ; \mathcal{K}_{\Gamma}\right) .
$$

If $x \in P$, then $x=\partial(y)$ for some $y \in T H_{2}(W, M ; \mathcal{R})$ by Lemma 3.5. Thus $B \ell(x)=\left(i^{\#} \circ \beta_{\mathrm{rel}}\right)(y)$. For every $p \in P, B \ell(x)(p)=\left(i^{\#} \beta_{\mathrm{rel}}\right)(y)(p)=\beta_{\mathrm{rel}}(y)\left(i_{*}(p)\right)$ $=\beta_{\text {rel }}(y)(0)=0$. Therefore $x \in P^{\perp}$, and $P \subset P^{\perp}$. The proof for the case when $M=\partial W$ follows from [COT1, Theorem 4.4].

Remark 3.7. If $W$ has more than one boundary component, then in general $P \neq P^{\perp}$. For example, if $W=M \times[0,1]$, then $P=0$ and $P^{\perp}=H_{1}(M ; \mathcal{R})$.

Suppose $\Gamma$ is a PTFA group with $H_{1}(\Gamma) \cong \Gamma /[\Gamma, \Gamma] \cong \mathbb{Z}$. Then its commutator subgroup, $[\Gamma, \Gamma]$, is also PTFA and $\mathbb{Z}[\Gamma, \Gamma]$ embeds into its (skew) quotient field of fractions denoted by $\mathbb{K}$. Then we have a PID $\mathbb{K}\left[t^{ \pm 1}\right]$ such that $\mathbb{Z} \Gamma \subset \mathbb{K}\left[t^{ \pm 1}\right] \subset \mathcal{K}_{\Gamma}$ where $t$ is identified with the generator of $\Gamma /[\Gamma, \Gamma]$.

The following generalizes [CT, Theorem 6.4]. The proof is the same once equipped with Proposition 3.6.

Theorem 3.8. Let $M$ be zero surgery on a knot $K$ in $S^{3}$. Let $W$ be an n-cylinder with $M$ as one of its boundary components. Suppose $\Gamma$ is an $(n-1)$-solvable PTFA group with $H_{1}(\Gamma) \cong \mathbb{Z}$. Suppose $\phi: \pi_{1}(W) \longrightarrow \Gamma$ induces an isomorphism upon 
abelianization. Let $d \equiv \operatorname{rk}_{\mathbb{Q}} H_{1}\left(M_{\infty} ; \mathbb{Q}\right)$ where $M_{\infty}$ is the (universal) infinite cyclic cover of $M$. Then

$$
\operatorname{rk}_{\mathbb{K}} \text { Image }\left\{i_{*}: H_{1}\left(M ; \mathbb{K}\left[t^{ \pm 1}\right]\right) \longrightarrow H_{1}\left(W ; \mathbb{K}\left[t^{ \pm 1}\right]\right)\right\} \geq(d-2) / 2 \text { if } n>1,
$$

and this rank is at least $d / 2$ if $n=1$.

Proof. Let $\mathcal{R} \equiv \mathbb{K}\left[t^{ \pm 1}\right]$. By [COT1, Theorem 2.13], there exists a nonsingular Blanchfield linking form

$$
B \ell: H_{1}(M ; \mathcal{R}) \longrightarrow H_{1}(M ; \mathcal{R})^{\#} \equiv \overline{\operatorname{Hom}_{\mathcal{R}}\left(H_{1}(M ; \mathcal{R}), \mathcal{K}_{\Gamma} / \mathcal{R}\right)} .
$$

Let $P \equiv \operatorname{Ker}\left\{i_{*}: H_{1}(M ; \mathcal{R}) \longrightarrow H_{1}(W ; \mathcal{R})\right\}, Q \equiv \operatorname{Image}\left\{i_{*}: H_{1}(M ; \mathcal{R}) \longrightarrow\right.$ $\left.H_{1}(W ; \mathcal{R})\right\}$, and $\mathcal{A} \equiv H_{1}(M ; \mathcal{R})$. By Proposition 3.6, $P \subset P^{\perp}$ with respect to the Blanchfield linking form. This gives us a well-defined map $f: P \longrightarrow(\mathcal{A} / P)^{\#}$ induced from the Blanchfield linking form. Since the Blanchfield linking form is nonsingular, $f$ is a monomorphism. Hence $\operatorname{rk}_{\mathbb{K}} P \leq \operatorname{rk}_{\mathbb{K}}(\mathcal{A} / P)^{\#}$.

We claim that $\operatorname{rk}_{\mathbb{K}} \mathcal{M}=\operatorname{rk}_{\mathbb{K}}(\mathcal{M})^{\#}$ for every finitely generated $\mathcal{R}$-module $\mathcal{M}$. $\left(\right.$ Here $(\mathcal{M})^{\#} \equiv \overline{\operatorname{Hom}_{\mathcal{R}}\left(\mathcal{M}, \mathcal{K}_{\Gamma} / \mathcal{R}\right)}$. $)$ Since $\mathcal{R}$ is a PID, it is enough to show this for the case when $\mathcal{M}$ is a cyclic $\mathcal{R}$-module, i.e., $\mathcal{M}=\mathcal{R} / p(t)$ where $p(t) \in \mathcal{R}$. For this cyclic case, $(\mathcal{R} / p(t))^{\#} \cong \mathcal{R} / \bar{p}(t)$ where $\bar{p}(t)$ is obtained by taking the involution of $p(t)$. Since $\operatorname{rk}_{\mathbb{K}} \mathcal{R} / p(t)$ is the degree of $p(t)$ for every $p(t) \in \mathcal{R}$ and $p(t)$ and $\bar{p}(t)$ have the same degree, $\mathrm{rk}_{\mathbb{K}} \mathcal{R} / p(t)=\mathrm{rk}_{\mathbb{K}} \mathcal{R} / \bar{p}(t)$. Using this we can deduce that

$$
\mathrm{rk}_{\mathbb{K}} P \leq \mathrm{rk}_{\mathbb{K}}(\mathcal{A} / P)^{\#}=\operatorname{rk}_{\mathbb{K}} \mathcal{A} / P=\mathrm{rk}_{\mathbb{K}} \mathcal{A}-\mathrm{rk}_{\mathbb{K}} P \text {. }
$$

Hence $\operatorname{rk}_{\mathbb{K}} P \leq \frac{1}{2} \operatorname{rk}_{\mathbb{K}} \mathcal{A}$. Since $Q \cong \mathcal{A} / P$ as $\mathcal{R}$-modules, we have

$$
\mathrm{rk}_{\mathbb{K}} Q=\operatorname{rk}_{\mathbb{K}} \mathcal{A}-\mathrm{rk}_{\mathbb{K}} P \geq \operatorname{rk}_{\mathbb{K}} \mathcal{A}-\frac{1}{2} \operatorname{rk}_{\mathbb{K}} \mathcal{A}=\frac{1}{2} \operatorname{rk}_{\mathbb{K}} \mathcal{A} .
$$

Thus we only need to show $\operatorname{rk}_{\mathbb{K}} \mathcal{A} \geq d-2$ if $n>1$ and $\operatorname{rk}_{\mathbb{K}} \mathcal{A}=d$ if $n=1$.

If $n=1, \Gamma \cong \mathbb{Z}, \mathbb{K}=\mathbb{Q}$, and $\mathcal{R}=\mathbb{Q}\left[t^{ \pm 1}\right]$. In this case $\mathcal{A}$ is the rational Alexander module, which is $H_{1}\left(M_{\infty} ; \mathbb{Q}\right)$. Hence $\operatorname{rk}_{\mathbb{K}} \mathcal{A}=d$. Suppose $n>1$. Observe that $\mathcal{A}$ is obtained from $H_{1}\left(S^{3} \backslash K ; \mathcal{R}\right)$ by killing the $\mathcal{R}$-submodule generated by the longitude, say $\ell$. By [C, Corollary 4.8], $\operatorname{rk}_{\mathbb{K}} H_{1}\left(S^{3} \backslash K ; \mathcal{R}\right) \geq d-1$. Since $(t-1)_{*} \ell=0$ in $H_{1}\left(S^{3} \backslash K ; \mathcal{R}\right)$, the submodule generated by $\ell$ is isomorphic with $\mathbb{K}\left[t^{ \pm 1}\right] /(t-1) \cong \mathbb{K}$. Hence $\operatorname{rk}_{\mathbb{K}} \mathcal{A} \geq d-2$.

\section{Obstructions to $n$-SOlvequivalence}

We use the information about twisted homology groups obtained in Section 3 to get obstructions to $n$-solvequivalence. Our obstructions will be vanishing of von Neumann $\rho$-invariants. The von Neumann $\rho$-invariants were firstly used by Cochran-Orr-Teichner to give obstructions to $n$-solvability (see Theorem 4.2 and 4.6 in [COT1]). We begin by giving a very brief explanation about von Neumann $\rho$ invariants. For more details on von Neumann $\rho$-invariants, the readers are referred to Section 5 in [COT1] and Section 2 in [CT]. Let $M$ be a compact, oriented 3manifold. If we have a representation $\phi: \pi_{1}(M) \longrightarrow \Gamma$ for a group $\Gamma$, then the von Neumann $\rho$-invariant or reduced $L^{(2)}$-signature $\rho(M, \phi)(\in \mathbb{R})$ is defined. If $(M, \phi)=\partial(W, \psi)$ for some compact, oriented 4-manifold $W$ and a homomorphism $\psi: \pi_{1}(W) \longrightarrow \Gamma$, then we have $\rho(M, \phi)=\sigma_{\Gamma}^{(2)}(W, \psi)-\sigma_{0}(W)$ where $\sigma_{\Gamma}^{(2)}(W, \psi)$ is the $L^{(2)}$-signature of the intersection form defined on $H_{2}(W ; \mathbb{Z} \Gamma)$ twisted by $\psi$ and $\sigma_{0}(W)$ is the ordinary signature of $W$. If $\Gamma$ is a PTFA group, then as we have seen before $\Gamma$ embeds into the (skew) quotient field of fractions $\mathcal{K}_{\Gamma}$, and $\sigma_{\Gamma}^{(2)}$ can be 
thought of as a homomorphism from $L^{0}\left(\mathcal{K}_{\Gamma}\right)$ to $\mathbb{R}$. Some useful properties of von Neumann $\rho$-invariants due to COT are given below. One can find detailed proofs or explanations in [COT1, Section 5].

Proposition 4.1. Let $M$ be as above and $\Gamma$ a PTFA group. Suppose we have a homomorphism $\phi: \pi_{1}(M) \longrightarrow \Gamma$.

(1) If $(M, \phi)=\partial(W, \psi)$ for some compact oriented 4 -manifold $W$ such that the equivariant intersection form on $\mathrm{H}_{2}\left(W ; \mathcal{K}_{\Gamma}\right)$ is nonsingular and admits a half-rank summand on which the form vanishes, then $\sigma_{\Gamma}^{(2)}(W, \psi)=0$. Thus if $\sigma_{0}(W)=0$, then $\rho(M, \phi)=0$.

(2) If $\phi$ factors through $\phi^{\prime}: \pi_{1}(M) \longrightarrow \Gamma^{\prime}$ where $\Gamma^{\prime}$ is a subgroup of $\Gamma$, then $\rho\left(M, \phi^{\prime}\right)=\rho(M, \phi)$.

(3) If $\phi$ is trivial, then $\rho(M, \phi)=0$.

(4) If $\Gamma=\mathbb{Z}$ and $\phi$ extends over $W$ nontrivially for some compact, spin 4manifold $W$, then $\rho(M, \phi)=\int_{\omega \in S^{1}} \sigma(h(\omega)) \mathrm{d} \omega-\sigma_{0}(W)$ where $h$ is the matrix representing the intersection form on $H_{2}\left(W ; \mathbb{C}\left[t, t^{-1}\right]\right) /($ torsion $)$.

Now we are ready to give obstructions to $n$-solvequivalence. In fact, we give a more general theorem.

Theorem 4.2. Let $M_{i}(i=1,2, \ldots, \ell)$ be closed, connected, oriented 3-manifolds with $H_{1}\left(M_{i} ; \mathbb{Q}\right) \cong \mathbb{Q}$ for all $i$. Let $\Gamma$ be an $n$-solvable group. Suppose there exists a rational (n.5)-cylinder $W$ with $\partial W=\coprod_{i=1}^{\ell} M_{i}$ (of arbitrary multiplicity) and we have representations $\phi_{i}: \pi_{1}\left(M_{i}\right) \longrightarrow \Gamma$. If there exists a homomorphism $\phi:$ $\pi_{1}(W) \rightarrow \Gamma$ inducing the homomorphisms $\phi_{i}$ for all $i$, then

$$
\sum_{i=1}^{\ell} \rho\left(M_{i}, \phi_{i}\right)=0 .
$$

The following is an easy corollary of Theorem 4.2; hence the proof is omitted.

Corollary 4.3. Suppose $K_{0}$ is (n.5)-solvequivalent to $K_{1}$ via $W$ and $\Gamma$ is an $n$ solvable group. Suppose we have representations $\phi_{i}: \pi_{1}\left(M_{i}\right) \longrightarrow \Gamma$ for $i=0,1$ where $M_{i}$ is zero surgery on $S^{3}$ along $K_{i}, i=0,1$. If there exists a homomorphism $\phi: \pi_{1}(W) \rightarrow \Gamma$ inducing the homomorphisms $\phi_{i}$ for $i=1,2$, then

$$
\rho\left(M_{0}, \phi_{0}\right)=\rho\left(M_{1}, \phi_{1}\right) .
$$

Proof of Theorem 4.2. Since $W$ is a $(0)$-cylinder, $\sigma_{0}(W)=0$ by Remark $2.2(1)$. Since $\sum_{i=1}^{\ell} \rho\left(M_{i}, \phi_{i}\right)=\sigma_{\Gamma}^{(2)}(W, \phi)-\sigma_{0}(W)$, we need only show that $\sigma_{\Gamma}^{(2)}(W, \phi)=0$. Let $\operatorname{rk}_{\mathbb{Q}}\left(H_{2}(W ; \mathbb{Q}) / I_{\mathbb{Q}}\right)=2 m$ where $\left.I_{\mathbb{Q}} \equiv \operatorname{Image}_{\text {Inc }_{*}}: H_{2}(\partial W ; \mathbb{Q}) \longrightarrow H_{2}(W ; \mathbb{Q})\right\}$. Let $L$ be a rational $(n+1)$-Lagrangian for $W$ generated by $(n+1)$-surfaces $\left\{\ell_{1}, \ell_{2}\right.$, $\left.\ldots, \ell_{m}\right\}$. Since $\Gamma$ is $n$-solvable, $\phi$ factors through $\pi_{1}(W) / \pi_{1}(W)^{(n+1)}$. Thus if we let $\mathcal{K}_{\Gamma}$ denote the (skew) quotient field of fractions of $\mathbb{Q} \Gamma$, then we can take the image of $L$ in $H_{2}\left(W ; \mathcal{K}_{\Gamma}\right)$, which is denoted by $L^{\prime}$ (generated by $\left\{\ell_{1}^{\prime}, \ell_{2}^{\prime}, \ldots, \ell_{m}^{\prime}\right\}$ ). By naturality of the intersection form, the intersection form $\lambda^{\prime}: H_{2}\left(W ; \mathcal{K}_{\Gamma}\right) \times$ $H_{2}\left(W ; \mathcal{K}_{\Gamma}\right) \rightarrow \mathcal{K}_{\Gamma}$ vanishes on $L^{\prime}$. Moreover the intersection form is nonsingular since $H_{*}\left(\partial W ; \mathcal{K}_{\Gamma}\right)=0$ by Corollary 3.3 . By Proposition $3.4, \operatorname{rk}_{\mathcal{K}_{\Gamma}} H_{2}\left(W ; \mathcal{K}_{\Gamma}\right)=$ $2 m$. Therefore if we show that $\left\{\ell_{1}^{\prime}, \ell_{2}^{\prime}, \ldots, \ell_{m}^{\prime}\right\}$ is linearly independent in $H_{2}\left(W ; \mathcal{K}_{\Gamma}\right)$, then $\operatorname{rk}_{\mathcal{K}_{\Gamma}} L^{\prime}=m=\frac{1}{2} \operatorname{rk}_{\mathcal{K}_{\Gamma}} H_{2}\left(W ; \mathcal{K}_{\Gamma}\right)$, which implies that $\sigma_{\Gamma}^{(2)}(W, \phi)=0$ by Proposition 4.1(1). 
For convenience we let $M \equiv \coprod_{i=2}^{\ell} M_{i}$ and $S \equiv\left\{\ell_{1}, \ell_{2}, \ldots, \ell_{m}\right\}$. Since $S$ generates a rational $(n+1)$-Lagrangian and $\#(S)=m=\frac{1}{2} \operatorname{rk}_{\mathbb{Q}}\left(H_{2}(W ; \mathbb{Q}) / I_{\mathbb{Q}}\right)$, the image of $S$ in $H_{2}(W ; \mathbb{Q}) / I_{\mathbb{Q}}$ is linearly independent. By investigating the long exact sequences of homology groups of the pairs $(W, \partial W)$ and $(W, M)$, one easily sees that $I_{\mathbb{Q}}=$ Image $\left\{\right.$ inc $\left._{*}: H_{2}(M ; \mathbb{Q}) \longrightarrow H_{2}(W ; \mathbb{Q})\right\}$. It follows that $H_{2}(W ; \mathbb{Q}) / I_{\mathbb{Q}}$ can be identified with a $\mathbb{Q}$-subspace of $H_{2}(W, M ; \mathbb{Q})$. Hence the image of $S$ in $H_{2}(W, M ; \mathbb{Q})$ is linearly independent. By the second part of Proposition 3.4, the image of $S$ in $H_{2}(W, M ; \mathbb{Q} \Gamma)$ is linearly independent. Hence the image of $S$ in $H_{2}\left(W, M ; \mathcal{K}_{\Gamma}\right)$ is also linearly independent. By Corollary $3.3, H_{1}\left(M ; \mathcal{K}_{\Gamma}\right)=H_{2}\left(M ; \mathcal{K}_{\Gamma}\right)=0$. This implies that $H_{2}\left(W ; \mathcal{K}_{\Gamma}\right) \cong H_{2}\left(W, M ; \mathcal{K}_{\Gamma}\right)$. Therefore $\left\{\ell_{1}^{\prime}, \ell_{2}^{\prime}, \ldots, \ell_{m}^{\prime}\right\}$ is linearly independent in $H_{2}\left(W ; \mathcal{K}_{\Gamma}\right)$.

\section{MAIN THEOREM}

In this section we prove the main theorem, that simultaneously generalizes the main theorems of $[\mathrm{CT}]$ and of $[\mathrm{L}],[\mathrm{K}]$. The strength of our theorem lies in being able to work within the class of knots that have the same fixed classical Seifert matrix (and Alexander module) and the same higher-order analogues of these. Therefore, after stating the theorem, we review the higher-order Alexander modules. Then we state and prove two theorems that are used at the end of the section to prove the main theorem. The first of these, Theorem 5.13, is an important technical result that significantly generalizes [CT, Theorem 4.3]. Due to its difficult technical nature, the proof of our Theorem 5.13 is not completed until the next section.

Theorem 5.1 (Main Theorem). Let $n \in \mathbb{N}$. Let $K$ be a knot whose Alexander polynomial has degree greater than 2 (if $n=1$, then degree equal to 2 is allowed). Then there is an infinite family of knots $\left\{K_{i} \mid i \in \mathbb{N}_{0}\right\}$ with $K_{0}=K$ such that:

(1) For each $i, K_{i}-K$ is n-solvable. In particular, $K_{i}$ is $n$-solvequivalent to $K$. Moreover, $K_{i}$ and $K$ cobound, in $S^{3} \times[0,1]$, a smoothly embedded symmetric Grope of height $n+2$.

(2) If $i \neq j, K_{i}$ is not (n.5)-solvequivalent to $K_{j}$. In particular, $K_{i}-K_{j}$ is not (n.5)-solvable, and $K_{i}$ and $K_{j}$ do not cobound, in $S^{3} \times[0,1]$, any embedded symmetric Grope of height $(n+2.5)$.

(3) Each $K_{i}$ has the same $m$-th integral higher-order Alexander module as $K$, for $m=0,1, \ldots, n-1$. Indeed, if $G_{i}$ and $G$ denote the knot groups of $K_{i}$ and $K$, respectively, then there is an isomorphism $G_{i} /\left(G_{i}\right)^{(n+1)} \rightarrow G / G^{(n+1)}$ that preserves the peripheral structures.

(4) Each $K_{i}$ has the same $m^{\text {th }}$-order Seifert presentation as $K$, for $m=$ $0,1, \ldots, n-1$.

(5) For each $i>j, K_{i}-K_{j}$ is of infinite order in $\mathcal{F}_{(n)} / \mathcal{F}_{(n .5)}$.

(6) If $i, j>0, s\left(K_{i}\right)=s\left(K_{j}\right)$ and $\tau\left(K_{i}\right)=\tau\left(K_{j}\right)$, where $s$ is Rasmussen's smooth concordance invariant and $\tau$ is the smooth concordance invariant of P. Ozsváth and Z. Szabó ([Ra], [OS]).

Since there certainly exist knots whose Alexander polynomial has degree 4, from (5) above we recover the result of Cochran and Teichner.

Corollary $5.2([\mathrm{CT}])$. For every positive integer $n, \mathcal{F}_{(n)} / \mathcal{F}_{(n .5)}$ has positive rank. 
Corollary 5.3. Let $n$ be a positive integer.

1. $\mathcal{G}_{n+2}^{\text {smooth }} / \mathcal{G}_{n+2.5}^{\text {smooth }}$ has elements of infinite order represented by knots $K$ with $s(K)=0$ and $\tau(K)=0$.

2. The kernel of the homomorphism $S T: \mathcal{G}_{n+2}^{\text {smooth }} \rightarrow \mathbb{Z} \times \mathbb{Z}$ given by $S T(K)=$ $(s(K), \tau(K))$ is infinite.

Proof of Corollary 5.3. For fixed $n$, consider the knot $K_{2}-K_{1}$, that is, $K_{2} \#\left(-K_{1}\right)$. Note that, by part (6) above, $\tau\left(K_{2}-K_{1}\right)=s\left(K_{2}-K_{1}\right)=0$. By part (1) above (applied first to $K_{1}$ and $K$ and then to $K_{2}$ and $K$ ), $K_{1}$ and $K_{2}$ cobound, in $S^{3} \times[0,1]$, a smoothly embedded symmetric Grope of height $n+2$. It follows that $K_{1} \#\left(-K_{2}\right)$ bounds a smoothly embedded symmetric Grope of height $n+2$ in $B^{4}$. This is seen by choosing an embedded arc from $K_{1}$ to $K_{2}$ in $S^{3} \times[0,1]$, contained in the first-stage surface of the Grope and avoiding the boundaries of the higher-stage surfaces. Deleting a tubular neighborhood of this arc, one gets the desired Grope in $B^{4}$. But if some multiple of $K_{2} \#\left(-K_{1}\right)$ were the boundary of a smoothly (or even topologically) embedded symmetric Grope of height $n+2.5$ in $B^{4}$, then by [COT1, Theorem 8.11], it would be of finite order in $\mathcal{F}_{(n)} / \mathcal{F}_{(n .5)}$ contradicting part (5) above. This completes the proof of the first part of the corollary.

For the second part of the corollary, just consider multiples of $K_{2}-K_{1}$. Alternatively, note that the analysis above applies equally well to any of the infinite set of $\operatorname{knots}\left\{K_{i}-K_{1}, i \geq 1\right\}$.

The higher-order Alexander modules of a knot were defined by the first author, and we give brief explanations here. For more details refer to [C].

Definition $5.4([\mathrm{C}])$. Let $n$ be a nonnegative integer. Let $K$ be a knot and $G \equiv$ $\pi_{1}\left(S^{3} \backslash K\right)$. The $n$-th (integral) higher-order Alexander module, $\mathcal{A}_{n}^{\mathbb{Z}}(K)$, of a knot $K$ is the first (integral) homology group of the covering space of $S^{3} \backslash K$ corresponding to $G^{(n+1)}$, considered as a right $\mathbb{Z}\left[G / G^{(n+1)}\right]$-module, i.e., $G^{(n+1)} / G^{(n+2)}$ as a right module over $\mathbb{Z}\left[G / G^{(n+1)}\right]$.

Using the local coefficient system $\pi: G \rightarrow G / G^{(n+1)}$, one can see that $\mathcal{A}_{n}^{\mathbb{Z}}(K) \cong$ $H_{1}\left(S^{3} \backslash K ; \mathbb{Z}\left[G / G^{(n+1)}\right]\right)$ as right $\mathbb{Z}\left[G / G^{(n+1)}\right]$-modules. Notice that $\mathcal{A}_{0}^{\mathbb{Z}}(K)$ is the classical Alexander module of $K$. Let $\Gamma_{n} \equiv G / G^{(n+1)}$. Let $\Gamma_{n}^{\prime}$ denote the commutator subgroup of $\Gamma_{n}$ (that is, $\left.G^{(1)} / G^{(n+1)}\right)$. Note that when $n=0, \Gamma_{n}^{\prime}=$ $\{e\}$. Let $\Sigma$ be a Seifert surface for $K$, let $E_{K} \equiv S^{3} \backslash K$ and let $Y \equiv E_{K}-(\Sigma \times(-1,1))$. We denote the two inclusions $\Sigma \rightarrow \Sigma \times\{ \pm 1\} \rightarrow \partial Y \subset Y$ by $i_{+}$and $i_{-}$. Notice that the coefficient system $\pi$, when restricted to $\pi_{1}(\Sigma)$ or $\pi_{1}(Y)$, has image in $\Gamma_{n}^{\prime}$. Thus $\Sigma$ and $Y$ have naturally induced $\mathbb{Z} \Gamma_{n}^{\prime}$-local coefficient systems and so we have the inclusion-induced maps $\left(i_{ \pm}\right)_{*}: H_{1}\left(\Sigma ; \mathbb{Z} \Gamma_{n}^{\prime}\right) \rightarrow H_{1}\left(Y ; \mathbb{Z} \Gamma_{n}^{\prime}\right)$. In the classical case $n=0$ these are just the usual maps $\left(i_{ \pm}\right)_{*}: H_{1}(\Sigma ; \mathbb{Z}) \rightarrow H_{1}(Y ; \mathbb{Z})$ that determine the Seifert matrix and Seifert form. For $n>0$, no analogue of the Seifert form has been found, so these maps, though quite complicated, play a crucial role. In the absence of a true analogue of a higher-order Seifert form they contain the relevant (integral) information necessary to reconstruct the Alexander modules (but, as in the classical case, contain more information than the modules alone). Therefore we make the following definition.

Definition 5.5. An $n^{\text {th }}$-order Seifert presentation of a knot $K$ is the ordered pair $\left(\left(i_{+}\right)_{*},\left(i_{-}\right)_{*}\right)$ of maps $\left(i_{ \pm}\right)_{*}: H_{1}\left(\Sigma ; \mathbb{Z} \Gamma_{n}^{\prime}\right) \rightarrow H_{1}\left(Y ; \mathbb{Z} \Gamma_{n}^{\prime}\right)$ induced by the inclusion maps $\left(i_{ \pm}\right): \Sigma \rightarrow Y$ for some choice of Seifert surface $\Sigma$ for $K$. 
Therefore, the case $n=1$ of our main theorem precisely recovers that of [Li] and the second author $[\mathrm{K}]$. In general, since $H_{1}\left(\Sigma ; \mathbb{Z} \Gamma_{n}^{\prime}\right)$ and $H_{1}\left(Y ; \mathbb{Z} \Gamma_{n}^{\prime}\right)$ may not be free modules (or even finitely generated), we cannot speak of a Seifert matrix. However after some localization this situation can be remedied.

Recall from [COT1], [C] that there is a localized version of $\mathcal{A}_{n}^{\mathbb{Z}}(K)$. We set $S_{n} \equiv \mathbb{Z} \Gamma_{n}^{\prime}-\{0\}, n \geq 0$. Then $S_{n}$ is a right divisor set of $\mathbb{Z} \Gamma_{n}$ and we let $\mathcal{R}_{n} \equiv$ $\left(\mathbb{Z} \Gamma_{n}\right)\left(S_{n}\right)^{-1}$. It also follows that $\mathbb{Z} \Gamma_{n}^{\prime}$ is itself an Ore domain and embeds into its classical (skew) quotient field of fractions, which we denote by $\mathbb{K}_{n}$. By [COT1, Proposition 3.2], $\mathcal{R}_{n}$ is canonically identified with the (skew) Laurent polynomial ring $\mathbb{K}_{n}\left[t^{ \pm}\right]$, which is a PID. In the classical case, $S_{0}=\mathbb{Z}-\{0\}, \mathbb{K}_{0}=\mathbb{Q}$ and $\mathcal{R}_{0}$ is $\mathbb{Q}\left[t^{ \pm 1}\right]$. In general, Cochran defines the localized higher-order Alexander module $[\mathrm{C}]$.

Definition 5.6. The $n$-th localized Alexander module of a knot $K$ is $\mathcal{A}_{n}(K) \equiv$ $H_{1}\left(S^{3} \backslash K ; \mathcal{R}_{n}\right)$.

Therefore the case $n=0$ gives the classical (rational) Alexander module. Moreover the $n^{t h}$-order Seifert presentation maps $\left(i_{ \pm}\right)_{*}: H_{1}\left(\Sigma ; \mathbb{Z} \Gamma_{n}^{\prime}\right) \rightarrow H_{1}\left(Y ; \mathbb{Z} \Gamma_{n}^{\prime}\right)$ determine their localized counterparts $\left(i_{ \pm}\right)_{*}: H_{1}\left(\Sigma ; \mathbb{K}_{n}\right) \rightarrow H_{1}\left(Y ; \mathbb{K}_{n}\right)$. The following proposition is due to Cochran and Harvey (for example, see [C, Proposition $6.1])$.

Proposition 5.7. The following sequence is exact:

$$
H_{1}\left(\Sigma ; \mathbb{K}_{n}\right) \otimes_{\mathbb{K}_{n}} \mathbb{K}_{n}\left[t^{ \pm 1}\right] \stackrel{d}{\rightarrow} H_{1}\left(Y ; \mathbb{K}_{n}\right) \otimes_{\mathbb{K}_{n}} \mathbb{K}_{n}\left[t^{ \pm 1}\right] \rightarrow \mathcal{A}_{n}(K) \rightarrow 0
$$

where $d(\alpha \otimes 1)=\left(i_{+}\right)_{*} \alpha \otimes t-\left(i_{-}\right)_{*} \alpha \otimes 1$.

Corollary 5.8 ([C, Corollary 6.2]). If the classical Alexander module of $K$ is not 1 , then $\mathcal{A}_{n}(K), n>0$, has a square presentation matrix of size $r=\max \{0,-\chi(\Sigma)\}$ each entry of which is a Laurent polynomial of degree at most 1 . Specifically, we have the presentation

$$
\left(\mathbb{K}_{n}\left[t^{ \pm 1}\right]\right)^{r} \stackrel{\partial}{\rightarrow}\left(\mathbb{K}_{n}\left[t^{ \pm 1}\right]\right)^{r} \rightarrow \mathcal{A}_{n}(K) \rightarrow 0
$$

where $\partial$ arises from the above proposition. If $n=0$, then the same holds with $r$ replaced by $\beta_{1}(\Sigma)$.

Definition 5.9. The above presentation matrix is called an order $n$ localized Seifert presentation matrix for $K$.

Proposition 5.10. Theorem 5.1 is false for $n \geq 2$ if the hypothesis on the degree of the Alexander polynomial is weakened to allow knots whose Alexander polynomials have degree 2.

Proof. Let $K$ denote the knot shown in Figure 1, well known as the "simplest" ribbon knot.

We claim that the conclusions of Theorem 5.1, for $n \geq 2$ are false for $K$ because, loosely speaking, any knot with the same classical Alexander module and first higher-order Alexander module as $K$ is topologically slice by recent work of Friedl and Teichner. Details follow.

One easily checks from direct calculation that if $\Delta$ is one of the two obvious ribbon disks obtained by "cutting one of the bands" in the figure, then $G=$ $\pi_{1}\left(B^{4}-\Delta\right)$ is isomorphic to $\mathbb{Z}\left[\frac{1}{2}\right] \rtimes \mathbb{Z}$ and in particular is 1-solvable, that is, $G^{(2)}=0$. 


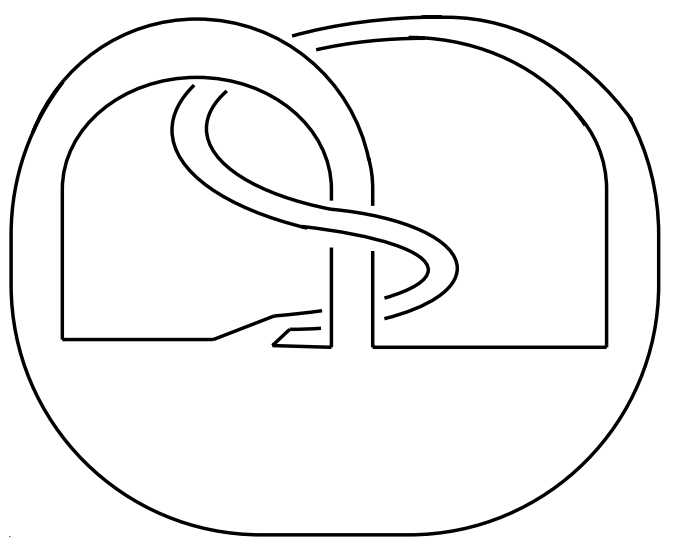

Figure 1

Let $P$ denote $\pi_{1}\left(M_{K}\right)$ where $M_{K}$ is the zero surgery on $K$. Then the inclusion map induces an epimorphism $\phi: P \rightarrow G$. Since $G^{(3)}=0$, this factors through $P / P^{(3)}$.

Suppose the conclusions of the main theorem were true for $K=K_{0}$ and let $K_{1}$ denote a knot other than $K$ that satisfies the conclusions for some $n \geq 2$. By property (1) of Theorem 5.1, $K_{1}$ is (0)-solvable and hence has Arf invariant zero (this also follows from property (4)). Let $P_{1}$ denote $\pi_{1}\left(M_{K_{1}}\right)$ where $M_{K_{1}}$ is the zero surgery on $K_{1}$. As a consequence of property (3), there is an isomorphism $f: P_{1} /\left(P_{1}\right)^{(3)} \rightarrow P / P^{(3)}$ (since the conjugacy classes of the longitudes correspond under the isomorphism given by (3)). Hence there is an epimorphism $\phi_{1}: P_{1} \rightarrow G$ factoring through $f$ (essentially $\phi \circ f$ ). Recall that for any coefficient system $\psi$ : $Q \rightarrow G$, where $Q$ is a group, we have the isomorphism

$$
H_{1}(Q ; \mathbb{Z} G) \cong \frac{\operatorname{ker} \psi}{[\operatorname{ker} \psi, \operatorname{ker} \psi]}
$$

and so in particular

$$
H_{1}\left(P_{1} ; \mathbb{Z} G\right) \cong \frac{\operatorname{ker} \phi_{1}}{\left[\operatorname{ker} \phi_{1}, \operatorname{ker} \phi_{1}\right]} \text { and } H_{1}(P ; \mathbb{Z} G) \cong \frac{\operatorname{ker} \phi}{[\operatorname{ker} \phi, \operatorname{ker} \phi]} .
$$

Since $G^{(2)}=0,\left(P_{1}\right)^{(2)} \subset \operatorname{ker} \phi_{1}$ and so $\left(P_{1}\right)^{(3)} \subset\left[\operatorname{ker} \phi_{1}, \operatorname{ker} \phi_{1}\right]$, and similarly for $P$. It follows that

$$
H_{1}\left(P_{1} ; \mathbb{Z} G\right) \cong H_{1}\left(P_{1} /\left(P_{1}\right)^{(3)} ; \mathbb{Z} G\right) \cong H_{1}\left(P /(P)^{(3)} ; \mathbb{Z} G\right) \cong H_{1}(P ; \mathbb{Z} G) .
$$

Since $K_{1}$ and $K$ are nontrivial, $M_{K_{1}}$ and $M_{K}$ are aspherical and so

$$
H_{1}\left(M_{K_{1}} ; \mathbb{Z} G\right) \cong H_{1}\left(P_{1} ; \mathbb{Z} G\right) \text { and } H_{1}\left(M_{K} ; \mathbb{Z} G\right) \cong H_{1}(P ; \mathbb{Z} G) \text {. }
$$

In summary, $H_{1}\left(M_{K_{1}} ; \mathbb{Z} G\right) \cong H_{1}\left(M_{K} ; \mathbb{Z} G\right)$, and thus it follows from [FT, Theorem 1.3] that $K_{1}$ is a (topologically) slice knot, contradicting property (2) of Theorem 5.1.

We explain our method to construct desired examples. This technique is called genetic modification which results in a satellite of a knot. One can find a detailed explanation of genetic modification in [COT2]. We briefly review this construction. Let $K$ be a knot and $\left\{\eta_{1}, \eta_{2}, \ldots, \eta_{m}\right\}$ be an oriented trivial link in $S^{3}$ which misses $K$. Suppose $\left\{J_{1}, J_{2}, \ldots, J_{m}\right\}$ is an $m$-tuple of auxiliary knots. For each $\eta_{i}$, remove a 
tubular neighborhood of $\eta_{i}$ in $S^{3}$ and glue in the exterior of a tubular neighborhood of $J_{i}$ along their common boundary, which is a torus, in such a way that the longitude of $\eta_{i}$ is identified with the meridian of $J_{i}$ and the meridian of $\eta_{i}$ with the longitude of $J_{i}$. The resulting knot is denoted by $K\left(\eta_{i}, J_{i}\right)$ and called the result of genetic modification performed on the seed knot $K$ with the infection $J_{i}$ along the axis $\eta_{i}$. This construction can also be described in the following way. For each $\eta_{i}$, take an embedded disk in $S^{3}$ bounded by $\eta_{i}$ such that it meets with $K$ transversally. Cut off $K$ along the disk, grab the cut strands of $K$, and tie them into the knot $J_{i}$ with 0 -framing as in Figure 2.

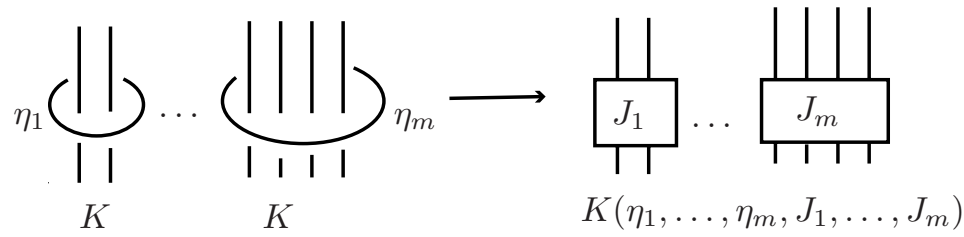

Figure 2. $K\left(\eta_{1}, \ldots, \eta_{m}, J_{1}, \ldots, J_{m}\right)$ : Genetic modification of $K$ by $J_{i}$ along $\eta_{i}$

Compare the following proposition with [COT2, Proposition 3.1].

Proposition 5.11. Let $K$ be a knot and $M$ be zero surgery on $K$. Suppose $n \geq 1$. Suppose $\left[\eta_{i}\right] \in \pi_{1}(M)^{(n)}$ and $J_{i}$ has vanishing Arf invariant for $i=1,2, \ldots, m$. Then $K$ is n-solvequivalent to $K^{\prime} \equiv K\left(\eta_{i}, J_{i}\right)$.

Proof. Since the $J_{i}$ have vanishing Arf invariant, they are 0 -solvable (see [COT1, Remark 1.3.2]). Let $W_{i}$ be a 0 -solution for $J_{i}$. By doing surgery along $\pi_{1}\left(W_{i}\right)^{(1)}$ we may assume $\pi_{1}\left(W_{i}\right) \cong \mathbb{Z}$, generated by the meridian of $J_{i}$.

Denote zero surgery on $K^{\prime}$ in $S^{3}$ by $M^{\prime}$. We construct an $n$-cylinder between $M$ and $M^{\prime}$. Take $M \times[0,1]$. Note that $\partial W_{i}=M_{i}=\left(S^{3} \backslash N\left(J_{i}\right)\right) \cup_{S^{1} \times S^{1}} S^{1} \times D^{2}$ where $N\left(J_{i}\right)$ denotes a tubular neighborhood of $J_{i}$ in $S^{3}$ and $\{*\} \times D^{2}$ in $S^{1} \times D^{2}$ denotes the surgery disk. Take the union of $M \times[0,1]$ and the $W_{i}$ 's in such a way that we identify a product neighborhood of $\eta_{i} \times\{1\}$ in $M \times\{1\}$, with the $i^{t h}$ copy of $S^{1} \times D^{2}$ in $\partial W_{i}$ for each $i$. We denote the 4-manifold resulting from this construction by $W$. One easily sees that $\partial W=M \amalg-M^{\prime}$. We claim that $W$ is an $n$-cylinder. Using a Mayer-Vietoris sequence $\left(M \times[0,1]\right.$ union $W_{i}$ intersecting along $\left.\eta_{i} \times D^{2}\right)$, one easily sees that $H_{1}(W) \cong \mathbb{Z}$ and the inclusions from $M$ to $W$ and $M^{\prime}$ to $W$ induce isomorphisms on the first homology. Also from the Mayer-Vietoris sequence one observes that $H_{2}(W) \cong H_{2}(M) \oplus\left(\bigoplus_{i=1}^{m} H_{2}\left(W_{i}\right)\right) \cong H_{2}\left(M^{\prime}\right) \oplus\left(\bigoplus_{i=1}^{m} H_{2}\left(W_{i}\right)\right)$. Note that the generator of $H_{2}(M)$ under the map $H_{2}(M) \longrightarrow H_{2}(W)$ maps to the image of the generator of $H_{2}\left(M^{\prime}\right)$ under the map $H_{2}\left(M^{\prime}\right) \longrightarrow H_{2}(W)$ since they are represented by capped-off Seifert surfaces. Thus $H_{2}(W) / i_{*}\left(H_{2}(\partial W)\right) \cong \bigoplus_{i=1}^{m} H_{2}\left(W_{i}\right)$ where $i_{*}$ is the homomorphism induced from the inclusion $i: \partial W \longrightarrow W$. Recall that $\pi_{1}\left(W_{i}\right)(\cong \mathbb{Z})$ is generated by the meridian of $J_{i}$ which is identified with the longitude of $\eta_{i}$. Hence $\pi_{1}\left(W_{i}\right)$ maps into $\pi_{1}(W)^{(n)}$ since $\left[\eta_{i}\right]$ lies in $\pi_{1}(M)^{(n)}$ by hypothesis and $\pi_{1}(M)^{(n)}$ maps into $\pi_{1}(W)^{(n)}$. This implies that 0-surfaces in $W_{i}$ are $n$-surfaces in $W$. Now by naturality of intersection forms, one can see that the union of the 0-Lagrangians (with 0 -duals for $\left.W_{i}\right)(0 \leq i \leq m)$ constitutes an $n$-Lagrangian (with $n$-duals) for $W$. One checks easily that $W$ is spin since each $W_{i}$ was spin. 
Suppose $K, \eta_{i}, J_{i}$ and $K^{\prime}$ are as in Proposition 5.11. Denote zero surgery on $J_{i}$ in $S^{3}$ by $M_{i}$. Suppose $W$ is the $n$-cylinder with $\partial W=M \amalg-M^{\prime}$ constructed above. Suppose $\psi: \pi_{1}(W) \longrightarrow \Gamma$ is a map to an arbitrary $n$-solvable PTFA group $\Gamma$. Let $\phi, \phi^{\prime}$, and $\phi_{i}$ denote the induced maps on $\pi_{1}(M), \pi_{1}\left(M^{\prime}\right)$, and $\pi_{1}\left(M_{i}\right)$ respectively. For each $J_{i}$, we denote by $\rho_{\mathbb{Z}}\left(J_{i}\right)$ the $\rho$-invariant $\rho\left(M_{i}, \zeta_{i}\right)$ where $\zeta_{i}: \pi_{1}\left(M_{i}\right) \longrightarrow \mathbb{Z}$ is the abelianization. The following lemma reveals the additivity of the $\rho$-invariant in our current setting.

\section{Lemma 5.12.}

$$
\rho(M, \phi)-\rho\left(M^{\prime}, \phi^{\prime}\right)=\sum_{i=1}^{m} \epsilon_{i} \rho_{\mathbb{Z}}\left(J_{i}\right)
$$

where $\epsilon_{i}=0$ or 1 according as $\phi\left(\eta_{i}\right)=$ e or not.

Proof. It follows from [COT2, Proposition 3.2] that

$$
\rho(M, \phi)-\rho\left(M^{\prime}, \phi^{\prime}\right)=\sum_{i=1}^{m} \rho\left(M_{i}, \phi_{i}\right) .
$$

Since $\pi_{1}\left(W_{i}\right) \cong \mathbb{Z}, \phi_{i}$ factors through $\mathbb{Z}$ generated by $\eta_{i}$. Thus its image is zero if $\phi\left(\eta_{i}\right)=e$, and $\mathbb{Z}$ if not (recall a PTFA group is torsion-free). In the former case, $\rho\left(M_{i}, \phi_{i}\right)=0$ by property (3) of Proposition 4.1. In the latter case, $\rho\left(M_{i}, \phi_{i}\right)=$ $\rho_{\mathbb{Z}}\left(J_{i}\right)$ by property $(2)$ of Proposition 4.1 .

The following two theorems are the key theorems that show the existence of a knot that is $n$-solvequivalent to a given knot $K$ but not (n.5)-solvequivalent to $K$. The first significantly generalizes [CT, Theorem 4.3] in two ways. Firstly, their theorem applies only when $K$ is a genus 2 fibered knot. Secondly their theorem only covers the case when $\partial W=M$. For a group $G$, let $G_{r}^{(k)}$ be the $k$-th rational derived group of $G$ (see [Ha, Section 3]).

Theorem 5.13. Let $n \in \mathbb{N}$. Let $K$ be a knot for which the degree of the Alexander polynomial is greater than 2 (if $n=1$, degree equal to 2 is allowed). Let $M$ be the zero framed surgery on $K$ in $S^{3}$. Suppose $\Sigma$ is a Seifert surface for $K$. Then there exists an oriented trivial link $\left\{\eta_{1}, \eta_{2}, \ldots, \eta_{m}\right\}$ in $S^{3}-\Sigma$ that satisfies the following:

(1) $\eta_{i} \in \pi_{1}(M)^{(n)}$ for all $i$. Moreover, the $\eta_{i}$ bound (smoothly embedded) symmetric capped gropes of height $n$, disjointly embedded in $S^{3}-K$ (except for the caps, which will hit $K$ ).

(2) For every $n$-cylinder $W$ with $M$ as one of its boundary components, there exists some $i$ such that $j_{*}\left(\eta_{i}\right) \notin \pi_{1}(W)_{r}^{(n+1)}$ where $j_{*}: \pi_{1}(M) \longrightarrow \pi_{1}(W)$ is induced from the inclusion $j: M \longrightarrow W$. The number of such i's is at least $\frac{1}{2}(d-2)$ if $n>1$ or at least $\frac{1}{2} d$ if $n=1$ where $d$ denotes the degree of the Alexander polynomial of $K$.

The proof of Theorem 5.13 is postponed to the end of Section 6 .

Let $c_{M}$ denote the universal bound for all $\rho$-invariants of a fixed 3-manifold $M$ given by [ChG, Theorem 4.10] and Ramachandran [R, Theorem 3.1.1]. That is, $|\rho(M, \phi)|<c_{M}$ for every representation $\phi: \pi_{1}(M) \longrightarrow \Gamma$ where $\Gamma$ is a group. For a detailed discussion see $[\mathrm{CT}]$. The final conclusion of the following theorem was proved in $[\mathrm{CT}]$ in the case that $K$ is itself an $n$-solvable knot. 
Theorem 5.14. Let $n \in \mathbb{N}$. Let $K$ be a knot in $S^{3}$. Suppose $\left\{\eta_{1}, \eta_{2}, \ldots, \eta_{m}\right\}$ is an oriented trivial link in $S^{3}$ that misses $K$ and has properties (1) and (2) of Theorem 5.13. Then, for every $m$-tuple $\left\{J_{1}, J_{2}, \ldots, J_{m}\right\}$ of Arf invariant zero knots for which $\rho_{\mathbb{Z}}\left(J_{i}\right)>2 c_{M}$, the knot $K^{\prime} \equiv K\left(\eta_{i}, J_{i}\right)$ (formed by genetic modification) is n-solvequivalent to $K$ but not (n.5)-solvequivalent to $K$. Moreover $K^{\prime}-K$ is of infinite order in $\mathcal{F}_{(n .0)} / \mathcal{F}_{(n .5)}$. (In particular, $K^{\prime} \#(-K)$ is $(n)$-solvable.)

Proof. By Proposition 5.11, $K^{\prime}$ is $n$-solvequivalent to $K$. Let $M$ be zero surgery on $K$ in $S^{3}$ and $M^{\prime}$ zero surgery on $K^{\prime}$ in $S^{3}$. Let $M_{i}$ be zero surgery on $J_{i}$ in $S^{3}$, $1 \leq i \leq m$. Let $W$ be an $n$-cylinder with $\partial W=M \amalg-M^{\prime}$ constructed as in the proof of Proposition 5.11. Suppose $M^{\prime}$ is $(n .5)$-solvequivalent to $M$ via $V$. We will show that this leads us to a contradiction. Let $X \equiv W \cup_{M^{\prime}} V$. Thus $\partial X=M \amalg-M$. We assume $\partial_{+} X=M$ and $\partial_{-} X=-M$. Since $V$ is an (n.5)-cylinder, it is an $n$-cylinder. As in Proposition 2.6, one can show that $X$ is an $n$-cylinder. Let $\Gamma \equiv \pi_{1}(X) / \pi_{1}(X)_{r}^{(n+1)}$, an $n$-solvable PTFA group. Let $\psi: \pi_{1}(X) \longrightarrow \Gamma$ be the projection. Let $\phi_{+}, \phi_{-}$, and $\phi^{\prime}$ denote the induced maps on $\pi_{1}\left(\partial_{+} X\right), \pi_{1}\left(\partial_{-} X\right)$, and $\pi_{1}\left(M^{\prime}\right)$, respectively. By Lemma 5.12 we have

$$
\rho\left(M, \phi_{+}\right)-\rho\left(M^{\prime}, \phi^{\prime}\right)=\sum_{i=1}^{m} \epsilon_{i} \rho_{\mathbb{Z}}\left(J_{i}\right)
$$

where $\epsilon_{i}=0$ or 1 according as $\phi_{+}\left(\eta_{i}\right)=e$ or not. On the other hand, since $V$ is an (n.5)-cylinder, Theorem 4.2 applies to say that

$$
\rho\left(M^{\prime}, \phi^{\prime}\right)-\rho\left(M, \phi_{-}\right)=0 .
$$

Hence

$$
\rho\left(M, \phi_{+}\right)-\rho\left(M, \phi_{-}\right)=\sum_{i=1}^{m} \epsilon_{i} \rho_{\mathbb{Z}}\left(J_{i}\right) .
$$

Note that since $X$ is an $n$-cylinder and the collection $\left\{\eta_{i}\right\}$ was chosen to satisfy property (2) of Theorem 5.13, there exists at least one $i$ such that $\psi\left(\eta_{i}\right) \neq e$, from which it follows that $\phi_{+}\left(\eta_{i}\right) \neq e$. Thus

$$
\rho\left(M, \phi_{+}\right)-\rho\left(M, \phi_{-}\right) \geq \rho_{\mathbb{Z}}\left(J_{i}\right)>2 c_{M},
$$

which is a contradiction. Therefore $K^{\prime}$ is not $(n .5)$-solvequivalent to $K$.

We will now show that $K^{\prime}-K \in \mathcal{F}_{(n)}$; that is, $K^{\prime} \#(-K)$ is $n$-solvable. Note that since $K^{\prime}$ was obtained from $K$ by genetic modification along the $\eta_{i}, K^{\prime} \#(-K)$ can be obtained from $K \#(-K)$ by performing a genetic modification along the "same" axes $\eta_{i}$. It is only necessary to observe that we can perform the connected sum in such a way as to preserve the fact that the $\eta_{i}$ lie in the $n^{\text {th }}$-derived group of $\pi_{1}$. This is clear because $S^{3}-K^{\prime}$ can be viewed as a subspace of $S^{3}-(K \#(-K))$. Notice that $K \#(-K)$ is a slice knot, hence $n$-solvable. By [COT2, Proposition 3.1], performing a genetic modification on an $n$-solvable knot using Arf invariant zero knots $J_{i}$, along axes that lie in the $n^{t h}$-derived group, results in another $n$-solvable knot. Hence $K^{\prime} \#(-K)$ is $n$-solvable.

Suppose $K^{\prime}-K$ were of order $k>0$ in $\mathcal{F}_{(n .0)} / \mathcal{F}_{(n .5)}$. We will show that this yields a contradiction, implying that $K^{\prime}-K$ is of infinite order. Our assumption is equivalent to the fact that $\left(\#_{i=1}^{k} K^{\prime}\right) \#\left(\#_{j=1}^{k}-K\right)$ is $(n .5)$-solvable. Let $N$ denote the zero surgery on $\left(\#_{i=1}^{k} K^{\prime}\right) \#\left(\#_{j=1}^{k}-K\right)$. Let $V$ be an (n.5)-solution for $N$ and let $W$ be as above. Let $M_{i}^{\prime}$ be the $i$-th copy of $M^{\prime}$ and $M_{j}$ be the $j$-th copy of $M, 1 \leq i, j \leq k$. Take a standard (spin) cobordism $C$ from $\left(\coprod_{i=1}^{k} M_{i}^{\prime}\right) \amalg$ 
$\left(\coprod_{j=1}^{k}-M_{j}\right)$ to $N$, which is obtained from $\left(\left(\coprod_{i=1}^{k} M_{i}^{\prime}\right) \amalg\left(\coprod_{j=1}^{k}-M_{j}\right)\right) \times[0,1]$ by adding $(2 k-1)$ 1-handles and then $(2 k-1)$ 2-handles (see [COT2, Lemma 4.2] for more details). Let $X \equiv\left(\coprod_{i=1}^{k} W_{i}\right) \cup_{\amalg M_{i}^{\prime}} C \cup_{N} V$ where $W_{i}$ is the $i$-th copy of $W$. Then $\partial X=\left(\coprod_{i=1}^{k} M_{i}\right) \amalg\left(\coprod_{j=1}^{k}-M_{j}\right)$. Let $\Gamma \equiv \pi_{1}(X) / \pi_{1}(X)_{r}^{(n+1)}$, an $n$-solvable PTFA group. Let $\psi: \pi_{1}(X) \longrightarrow \Gamma$ be the projection. Let $\phi_{j}, \phi_{i}^{\prime}$, $\phi_{i}^{+}$, and $\phi$ denote the restrictions of $\psi$ to $\pi_{1}\left(-M_{j}\right), \pi_{1}\left(M_{i}^{\prime}\right), \pi_{1}\left(M_{i}\right)$ (the upper boundary of $\left.W_{i}\right)$, and $\pi_{1}(N)$ respectively. One sees that $C$ is an (n.5)-cylinder since $H_{2}(C) / i_{*}\left(H_{2}(\partial C)\right)=0$. Thus by Theorem 4.2 ,

$$
\sum_{i=1}^{k} \rho\left(M_{i}^{\prime}, \phi_{i}^{\prime}\right)+\sum_{j=1}^{k} \rho\left(-M_{j}, \phi_{j}\right)-\rho(N, \phi)=0 .
$$

But since $V$ is an (n.5)-solution for $N, \rho(N, \phi)=0$ by Theorem 4.2. Therefore $\sum_{i=1}^{k} \rho\left(M_{i}^{\prime}, \phi_{i}^{\prime}\right)=\sum_{j=1}^{k} \rho\left(M_{j}, \phi_{j}\right)$. By Lemma 5.12 (applied for each $i$ and then summed),

$$
\sum_{i=1}^{k} \rho\left(M_{i}, \phi_{i}^{+}\right)-\sum_{i=1}^{k} \rho\left(M_{i}^{\prime}, \phi_{i}^{\prime}\right)=\sum_{i=1}^{k} \sum_{l=1}^{m} \epsilon_{i l} \rho_{\mathbb{Z}}\left(J_{l}\right)
$$

where $\epsilon_{i l}=0$ if $\phi_{i}^{+}\left(\eta_{i l}\right)=e$ and $\epsilon_{i l}=1$ if $\phi_{i}^{+}\left(\eta_{i l}\right) \neq e$. (By $\eta_{i l}$ we mean the $l$-th axis $\eta_{l}$ for the $i$-th copy of $K$.) Thus

$$
\sum_{i=1}^{k} \rho\left(M_{i}, \phi_{i}^{+}\right)-\sum_{j=1}^{k} \rho\left(M_{j}, \phi_{j}\right)=\sum_{i=1}^{k} \sum_{l=1}^{m} \epsilon_{i l} \rho_{\mathbb{Z}}\left(J_{l}\right) .
$$

Now we claim that $X$ is an $n$-cylinder. Using a Mayer-Vietoris sequence and [COT2, Lemma 4.2], one can show that $H_{2}(X) / i_{*}\left(H_{2}(\partial X)\right) \cong\left(\bigoplus_{i=1}^{k} H_{2}\left(W_{i}\right)\right) \oplus H_{2}(V)$ and the union of the 0-Lagrangians with 0-duals for $W_{i}(1 \leq i \leq k)$ and the $n$ Lagrangian with $n$-duals for $V$ constitutes an $n$-Lagrangian with $n$-duals for $X$. Since, for any $i, X$ is an $n$-cylinder with $M_{i}$ as one of its boundary components, by Theorem 5.13, for each $i$ there exists some $l_{i}$ such that $\psi\left(\eta_{i l_{i}}\right) \neq e$. Hence $\phi_{i}^{+}\left(\eta_{i l_{i}}\right) \neq e$. Then the right-hand side of the last equation is greater than $2 k c_{M}$, while the left-hand side is less than $2 k c_{M}$, a contradiction.

Finally we prove the main theorem.

Proof of Theorem 5.1 (Main Theorem). Let $K$ be a knot for which the degree of the Alexander polynomial is greater than 2 (if $n=1$, degree equal to 2 is allowed). Let $M$ denote the zero surgery on $K$. Apply Theorem 5.13 to choose an oriented trivial link $\left\{\eta_{1}, \eta_{2}, \ldots, \eta_{m}\right\}$ in $S^{3}$ that misses $K$ (except for the caps, which will hit $K$ ), and such that the $\eta_{i}$ bound height $n$ (smoothly embedded) symmetric capped gropes disjointly embedded in $S^{3}-K$. Let $c_{M}$ be the universal bound for $\rho$-invariants for $M$ as mentioned above. Choose knots $J^{i}(i \in \mathbb{N})$ with vanishing Arf invariants inductively as follows. First, choose $J^{1}$ such that $\rho_{\mathbb{Z}}\left(J^{1}\right)>2 c_{M}$. Suppose $J^{k-1}$ has been constructed. Then choose $J^{k}$ such that $\rho_{\mathbb{Z}}\left(J^{k}\right)>2 c_{M}+2 m \rho_{\mathbb{Z}}\left(J^{k-1}\right)$. These $J^{i}$ are easily found by taking the connected sum of a suitably large even number of copies of the left-handed trefoil. (Note that for the left-handed trefoil $J, \rho_{\mathbb{Z}}(J)=4 / 3$ by Proposition 4.1(4).) However, to achieve the final conclusion of Part (1), we must choose each $J^{i}$ to be a suitably large connected sum of the knot shown in [CT, Figure 3.6]. This knot has the same $\rho_{\mathbb{Z}}$ as the left-handed trefoil knot [CT, Lemma 4.5]. For each $i$, let $J_{j}^{i}$ be the $j$-th copy of $J^{i}, 1 \leq j \leq m$. Now 
define $K_{i} \equiv K\left(\eta_{1}, \ldots, \eta_{m}, J_{1}^{i}, \ldots, J_{m}^{i}\right)$, the result of genetic modification performed on $K$ with the infections $J_{j}^{i}$ along the axes $\eta_{j}(1 \leq j \leq m)$. Set $K_{0} \equiv K$.

Part (1) : It follows from the last sentence of Theorem 5.14 that $K_{i}-K \in \mathcal{F}_{(n)}$, $i>0$ and hence that $K_{i}$ is $n$-solvequivalent to $K$ (or apply Proposition 5.11). To show that $K_{i}$ and $K$ cobound, in $S^{3} \times[0,1]$, a (smoothly embedded) embedded symmetric Grope of height $n+2$, merely apply the proof of [CT, Theorem 3.8].

Part (2): Suppose $i>j \geq 0$. Let $M_{i}$ be the zero surgery on $K_{i}$ in $S^{3}$. Suppose $M_{i}$ is (n.5)-solvequivalent to $M_{j}$ via $U\left(\partial U=M_{i} \amalg-M_{j}\right)$. Suppose $V$ is an $n$-cylinder with $\partial V=M \amalg-M_{i}$ and $W$ an $n$-cylinder with $\partial W=M_{j} \amalg-M$ such that $V$ and $W$ are constructed as in the proof of Proposition 5.11. Let $X \equiv V \cup_{M_{i}} U \cup_{M_{j}} W$. Then $X$ is an $n$-cylinder with $\partial_{+} X=M$ and $\partial_{-} X=-M$ (see Proposition 2.6). Let $\Gamma \equiv \pi_{1}(X) / \pi_{1}(X)_{r}^{(n+1)}$, an $n$-solvable PTFA group. Let $\psi: \pi_{1}(X) \longrightarrow \Gamma$ be the projection. Let $\phi_{+}, \phi_{i}, \phi_{j}$, and $\phi_{-}$denote the restrictions of $\psi$ to $\pi_{1}\left(\partial_{+} X\right)$ $\left(=\pi_{1}(M)\right), \pi_{1}\left(M_{i}\right), \pi_{1}\left(M_{j}\right)$, and $\pi_{1}\left(\partial_{-} X\right)\left(=\pi_{1}(-M)\right)$ respectively. Since $U$ is an (n.5)-cylinder, by Corollary $4.3, \rho\left(M_{i}, \phi_{i}\right)=\rho\left(M_{j}, \phi_{j}\right)$. On the other hand, by Lemma 5.12,

$$
\rho\left(M, \phi_{+}\right)-\rho\left(M_{i}, \phi_{i}\right)=\sum_{k=1}^{m} \epsilon_{k} \rho_{\mathbb{Z}}\left(J_{k}^{i}\right)
$$

where $\epsilon_{k}=0$ if $\phi_{+}\left(\eta_{k}\right)=e$ and $\epsilon_{k}=1$ if $\phi_{+}\left(\eta_{k}\right) \neq e$. Similarly,

$$
\rho\left(M, \phi_{-}\right)-\rho\left(M_{j}, \phi_{j}\right)=\sum_{k=1}^{m} \epsilon_{k}^{\prime} \rho_{\mathbb{Z}}\left(J_{k}^{j}\right)
$$

where $\epsilon_{k}^{\prime}=0$ if $\phi_{-}\left(\eta_{k}\right)=e$ and $\epsilon_{k}^{\prime}=1$ if $\phi_{-}\left(\eta_{k}\right) \neq e$. Thus we have

$$
\rho\left(M, \phi_{+}\right)-\rho\left(M, \phi_{-}\right)=\sum_{k=1}^{m} \epsilon_{k} \rho_{\mathbb{Z}}\left(J_{k}^{i}\right)-\sum_{k=1}^{m} \epsilon_{k}^{\prime} \rho_{\mathbb{Z}}\left(J_{k}^{j}\right) .
$$

Since $J_{k}^{j}$ is merely a copy of $J^{j}, \rho_{\mathbb{Z}}\left(J_{k}^{j}\right)=\rho_{\mathbb{Z}}\left(J^{j}\right)$, and similarly $\rho_{\mathbb{Z}}\left(J_{k}^{i}\right)=\rho_{\mathbb{Z}}\left(J^{i}\right)$. Moreover, by Theorem 5.13 applied to $M=\partial_{+} X$, there is some $k$ such that $\phi_{+}\left(\eta_{k}\right)=\psi\left(\eta_{k}\right) \neq e$. Thus the right-hand side above is greater than $\rho_{\mathbb{Z}}\left(J^{i}\right)-$ $m \rho_{\mathbb{Z}}\left(J^{j}\right)$, which, by our choice of $J^{i}$ and $J^{j}$ is greater than $2 c_{M}$. This is a contradiction since the left-hand side above has absolute value less than $2 c_{M}$. To show that $K_{i}$ and $K_{j}$ do not cobound, in $S^{3} \times[0,1]$, an embedded symmetric Grope of height $(n+2.5)$, merely note that the proof of [COT1, Theorem 8.11] clearly applies to show that if they did bound such a Grope, then they would be (n.5)-solvequivalent. Part (3) : This follows from [C, Theorem 8.1].

Part (4) : We shall employ the terminology set out just above Proposition 5.7. Let $K_{*}$ denote one of the $K_{i}$ and let $G_{*}$ denote its knot group. Let $G$ denote the knot group of $K$. By part (3), these two knots share the same higher-order (integral) Alexander modules up to order $n-1$ and $G / G^{(i+1)}$ is isomorphic to $G_{*} /\left(G_{*}\right)^{(i+1)}$ for all $i \leq n$. Therefore in considering the $i^{t h}$ higher-order Alexander modules of these knots, we may consider that they are modules over the same ring, $\mathbb{Z} \Gamma_{i} \equiv \mathbb{Z}\left[G / G^{(i+1)}\right]$, as long as $i \leq n$. We shall show that there exist Seifert surfaces $\Sigma_{*}$ for $K_{*}$ and $\Sigma$ for $K$ with respect to which the $i^{t h}$-order Seifert presentations, $i_{ \pm}$: $H_{1}\left(\Sigma ; \mathbb{Z} \Gamma_{i}^{\prime}\right) \rightarrow H_{1}\left(Y ; \mathbb{Z} \Gamma_{i}^{\prime}\right)$ and $i_{ \pm}: H_{1}\left(\Sigma_{*} ; \mathbb{Z} \Gamma_{i}^{\prime}\right) \rightarrow H_{1}\left(Y_{*} ; \mathbb{Z} \Gamma_{i}^{\prime}\right)$, are identical up to isomorphisms identifying the domain and range of each, as long as $i \leq n-1$. (Recall that $\Gamma_{i}^{\prime}$ is the commutator subgroup of $\Gamma_{i}$.) A Seifert presentation determines the map $d$ in Proposition 5.7, and hence there are bases with respect to which the 
localized $i^{\text {th }}$-order Seifert matrices for $K_{*}$ and $K$ are identical. We may assume that $n \geq 1$.

Let $E(K)$ denote the exterior of $K$ in $S^{3}$. The continuous map $f: E\left(K_{*}\right) \rightarrow$ $E(K)$ that induces all of these isomorphisms is described as follows (see [C, Theorem 8.1]). Recall that $E\left(K_{*}\right)$ is constructed from $E(K)$ by replacing a collection of solid tori $\eta_{j} \times D^{2}$ by a collection of knot exteriors $E\left(J_{j}\right)$. Since it is well known that there is always a degree one map, $f_{j}$, relative to the boundary, from $E\left(J_{j}\right)$ to $E$ (unknot $) \equiv \eta_{j} \times D^{2}$, there is a degree one map relative to the boundary, $f$, from $E\left(K_{*}\right)$ to $E(K)$. Recall also that $\eta_{j} \in \pi(M)^{(n)}$ by choice. But, in fact, the $\eta_{j}$ actually produced by Theorem 6.4 lie in $F^{(n-1)}$ where $F \rightarrow \pi_{1}\left(M-\Sigma^{*}\right)$ where $\Sigma^{*}$ is a capped-off Seifert surface. Hence, the circles $\eta_{j}$ can be chosen to miss the given Seifert surface $\Sigma$ and actually represent elements of $\pi_{1}(Y)^{(n-1)} \subset G^{(n)}$. Therefore we can use the 'same' Seifert surface for $K_{*}$ as for $K$. For simplicity for the rest of this proof we shall assume that there is just one circle, $\eta$. The proof is no different for a collection. Note that $f$ is the 'identity' on a neighborhood of $\Sigma_{*}$ (mapping to $\Sigma)$ and restricts to a degree one map relative to the boundary $f: Y_{*} \rightarrow Y$. We need only show that this map carries $H_{1}\left(Y_{*} ; \mathbb{Z} \Gamma_{i}^{\prime}\right)$ isomorphically to $H_{1}\left(Y ; \mathbb{Z} \Gamma_{i}^{\prime}\right)$. Briefly, this is true because $\eta \in G^{(n)}$ and thus goes to zero in $\Gamma_{i}$ if $i \leq n-1$. It follows that the entire group $\pi_{1}(E(J))$, where $J$ is the infection knot, maps to zero in $\Gamma_{i}^{\prime}$. Thus in a Mayer-Vietoris analysis, $H_{1}\left(E(J) ; \mathbb{Z} \Gamma_{i}^{\prime}\right)$ really has untwisted coefficients and thus is not distinguishable from the case that $J$ is a trivial knot. But if $J$ were trivial, then $K_{*}=K$ and $Y_{*}=Y$. For more details, the proof is the same as the proof of [C, Theorem 8.2] where it is shown that each of $H_{1}\left(Y_{*} ; \mathbb{Z} \Gamma_{i}\right)$ and $H_{1}\left(Y ; \mathbb{Z} \Gamma_{i}\right)$ is isomorphic to the quotient of $H_{1}\left(Y \backslash\left(\eta \times D^{2}\right) ; \mathbb{Z} \Gamma_{i}\right)$ by the submodule generated by the meridian of $\eta$.

Part (5) : For $j=0$ this follows directly from Theorem 5.14. For general $j$ the proof is essentially the same as the proof of part (2). We outline the proof. Here $i$ and $j$ are fixed.

Let $M_{i}, M_{j}$, and $M$ denote the zero surgeries on $K_{i}, K_{j}$, and $K$ as usual. Suppose $K_{i}-K_{j}$ were of order $k>0$, that is to say, $\left(\#_{l=1}^{k} K_{i}\right) \#\left(\#_{l=1}^{k}-K_{j}\right)$ is (n.5)-solvable. Then by Proposition 2.7, $M_{\# K_{i}}$ is (n.5)-solvequivalent to $M_{\# K_{j}}$ via some $U$ as in the proof of part (2). Refer to the schematic Figure 3.

Now let $C$ be the standard cobordism between $M_{\# K_{i}}$ and the disjoint union of $k$ copies of $M_{i}$, which we denote by $M_{i}^{l}$ for $1 \leq l \leq k$, and let $D$ be the standard cobordism between $-M_{\# K_{j}}$ and the disjoint union of $k$ copies of $-M_{j}$, which we denote by $-M_{j}^{l}$ for $1 \leq l \leq k$. Let $V^{l}$ denote the $l^{\text {th }}$ copy of the standard $n$ cylinder between $M_{i}$ and $M$, constructed in the proof of Proposition 5.11, so that $\partial V^{l}=M^{l_{v}} \amalg-M_{i}^{l}$ where we use $M^{l_{v}}$ to denote the copy of $M$ that occurs in the boundary of $V^{l}$. Similarly let $W^{l}$ denote the $l^{\text {th }}$ copy of the standard $n$-cylinder between $-M_{j}$ and $-M$ so that $\partial W^{l}=M^{l_{w}} \amalg-M_{j}^{l}$ where we use $M^{l_{w}}$ to denote the copy of $M$ that occurs in the boundary of $W^{l}$. Following the proof of Part (2), let

$$
X \equiv\left(\coprod_{l=1}^{k} V^{l}\right) \cup C \cup_{M_{\# K_{i}}} U \cup_{M_{\# K_{j}}} D \cup\left(\coprod_{l=1}^{k} W^{l}\right) .
$$

Recall that we saw in the proof of Theorem 5.13 that $C$ and $D$ are (n.5)-cylinders since $H_{2}(C) / i_{*}\left(H_{2}(\partial C)\right)=0$ and $H_{2}(D) / i_{*}\left(H_{2}(\partial D)\right)=0$. It follows that $X$ is 


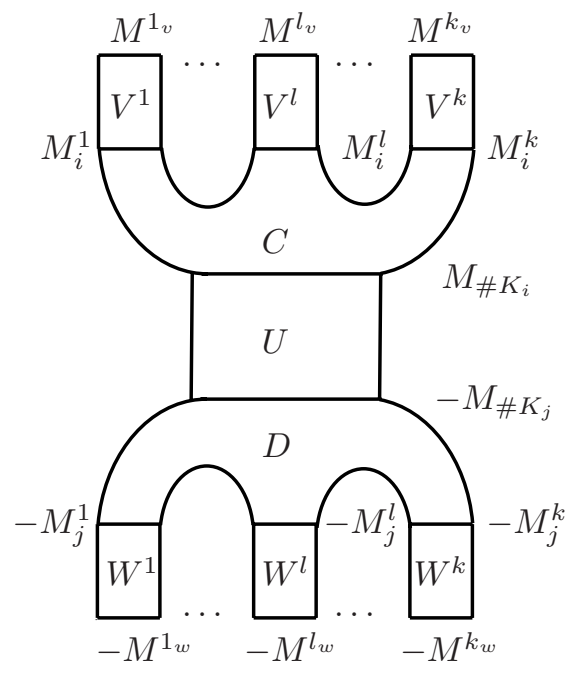

Figure 3

an $n$-cylinder with $\partial_{+} X=\coprod_{l=1}^{k} M^{l_{v}}$ and $\partial_{-} X=\coprod_{l=1}^{k}-M^{l_{w}}$ (see also Proposition 2.6). Let $\Gamma \equiv \pi_{1}(X) / \pi_{1}(X)_{r}^{(n+1)}$, an $n$-solvable PTFA group. Let $\psi$ : $\pi_{1}(X) \longrightarrow \Gamma$ be the projection. Let $\phi^{l_{v}}$ and $\phi^{l_{w}}$ denote the restrictions of $\psi$ to $\pi_{1}\left(M^{l_{v}}\right)$, and $\pi_{1}\left(M^{l_{w}}\right)$ respectively. Since $U$ is an (n.5)-cylinder and otherwise $H_{2}(X)$ comes from the $V^{l}$ and the $W^{l}$, we arrive at an expression similar to that of the proof of Part (2), except summed from $l=1$ to $k$ :

$$
\sum_{l=1}^{k}\left(\rho\left(M^{l_{v}}, \phi^{l_{v}}\right)-\rho\left(M^{l_{w}}, \phi^{l_{w}}\right)\right)=\sum_{l=1}^{k}\left(\sum_{s=1}^{m} \epsilon_{s l} \rho_{\mathbb{Z}}\left(J^{i}\right)-\sum_{s=1}^{m} \epsilon_{s l}^{\prime} \rho_{\mathbb{Z}}\left(J^{j}\right)\right),
$$

where $\epsilon_{s l}=0$ if $\phi^{l_{v}}\left(\eta_{s l}\right)=e$ (where $\eta_{s l}$ means the $s$-th axis $\eta_{s}$ for $K$ for constructing the $l$-th copy of $\left.K_{i}\right)$ and $\epsilon_{s l}=1$ if $\phi^{l_{v}}\left(\eta_{s l}\right) \neq e$, and where $\epsilon_{s l}^{\prime}=0$ if $\phi^{l_{w}}\left(\eta_{s l}^{\prime}\right)=e$ (where $\eta_{s l}^{\prime}$ means the $s$-th axis $\eta_{s}$ for $K$ for constructing the $l$-th copy of $K_{j}$ ) and $\epsilon_{s l}^{\prime}=1$ if $\phi^{l_{w}}\left(\eta_{s l}^{\prime}\right) \neq e$. By property (2) of Theorem 5.13 applied, for each $l$, to $M^{l_{v}}$, there is some $s$ such that $\phi^{l_{v}}\left(\eta_{s l}\right)=\psi\left(\eta_{s l}\right) \neq e$. Thus, for each $l$, the $l^{\text {th }}$ term of the summation on the right-hand side above is greater than $\rho_{\mathbb{Z}}\left(J^{i}\right)-m \rho_{\mathbb{Z}}\left(J^{j}\right)$, which, by our choice of $J^{i}$ and $J^{j}$ is greater than $2 c_{M}$. Thus the right-hand side is greater than $2 k c_{M}$. This is a contradiction since the left-hand side above has absolute value less than $2 k c_{M}$, since $M^{l_{v}} \cong M^{l_{w}} \cong M$.

Part (6) : Let $g$ be the genus of $K$. Note that in the construction of $K_{i}$ we could have chosen any Seifert surface for $K$, in particular one of genus $g$. Recall that the circles $\eta_{i}$ are chosen in the complement of this Seifert surface. Hence, by construction, genus $\left(K_{i}\right) \leq g$. It follows that $g_{s}(K) \leq g$, where $g_{s}$ denotes the smooth slice genus, and thus that $\left|s\left(K_{i}\right)\right| \leq 2 g$ and $\left|\tau\left(\bar{K}_{i}\right)\right| \leq g$ by [Ra] and [OS] respectively. Consider the function $S T$ from $\left\{K_{i}\right\}$ to $\mathbb{Z} \times \mathbb{Z}$ given by $S T\left(K_{i}\right)=$ $\left(s\left(K_{i}\right), \tau\left(K_{i}\right)\right)$. Since the image of this function is a finite set, there is a subsequence $K_{i_{j}}$ on which $S T$ is constant. Redefining our $K_{i}$ to be this subsequence gives the claimed result. 


\section{Algebraic $n$-SOlution}

The purpose of this section is to complete the proof of Theorem 5.13, which relies on Theorem 6.4. In this section we define and investigate an algebraic $n$ solution. One might think of this as an algebraic abstraction of an $n$-cylinder (or an $n$-solution). In [CT], Cochran and Teichner defined an algebraic $n$-solution. Our notion is much more general. In particular, an algebraic $n$-solution in [CT] is defined using only the free group of rank 4, but our version is defined using the free group of (arbitrary) even rank. Moreover, the proof of [CT] that a (geometric) $n$-solution induces an algebraic $n$-solution is valid only for fibered knots of genus 2 . For a group $G$, let $G_{k}$ denote $G / G_{r}^{(k)}$ where $G_{r}^{(k)}$ is the $k$-th rational derived group of $G$. Then, as noted before, $G_{k}$ is a $(k-1)$-solvable PTFA group and embeds into its skew quotient field of fractions, which is denoted by $\mathbb{K}\left(G_{k}\right)$.

Definition 6.1. Let $S$ be a group such that $H_{1}(S ; \mathbb{Q}) \neq 0$. Suppose $F \stackrel{i}{\longrightarrow} S$ is a fixed homomorphism from the free group of rank $2 g$. A nontrivial homomorphism $r: S \rightarrow G$ is called an algebraic $n$-solution $(n \geq 0$ ) (for $F \stackrel{i}{\longrightarrow} S$ ) if the following hold:

(1) For each $0 \leq k \leq n-1$ the image of the following composition, after tensoring with the quotient field $\mathbb{K}\left(G_{k}\right)$ of $\mathbb{Z} G_{k}$, is nontrivial:

$$
H_{1}\left(S ; \mathbb{Z} G_{k}\right) \stackrel{r_{*}}{\longrightarrow} H_{1}\left(G ; \mathbb{Z} G_{k}\right) \cong G_{r}^{(k)} /\left[G_{r}^{(k)}, G_{r}^{(k)}\right] \rightarrow G_{r}^{(k)} / G_{r}^{(k+1)} .
$$

(2) For each $0 \leq k \leq n$, the map $H_{1}\left(F ; \mathbb{Z} G_{k}\right) \stackrel{i_{*}}{\longrightarrow} H_{1}\left(S ; \mathbb{Z} G_{k}\right)$, after tensoring with the quotient field $\mathbb{K}\left(G_{k}\right)$, is surjective.

Remark 6.2. (1) If $n \geq 0$, then, by combining conditions (1) and (2) for $k=0$, we conclude that $H_{1}(F ; \mathbb{Q}) \longrightarrow H_{1}(G ; \mathbb{Q})$ is nontrivial. Thus, for some $i$, $r_{*}\left(x_{i}\right)$ is nontrivial in $G_{1}=G / G_{r}^{(1)}$ where $\left\{x_{1}, x_{2}, \ldots, x_{m}\right\}$ is a generating set for $F$.

(2) In all the applications the image of the map in (1) above has rank at least one half the rank of $H_{1}\left(S ; \mathbb{Z} G_{k}\right)$.

(3) If $r: S \rightarrow G$ is an algebraic $n$-solution then, for any $k<n$ it is an algebraic $k$-solution.

Of course we need to establish that the primary geometric examples do in fact satisfy the algebraic conditions above.

Proposition 6.3. Suppose $K$ is a knot for which the degree of the classical Alexander polynomial is greater than 2 (if $n=1$, then degree equal to 2 is allowed). Suppose $M$ is the zero surgery on $K$ in $S^{3}, \Sigma$ is a capped-off Seifert surface (of genus g) for $K$, and $S \equiv \pi_{1}(M)^{(1)}$. Suppose $F \rightarrow \pi_{1}(M-\Sigma)$ is any map inducing an isomorphism on $H_{1}(F ; \mathbb{Q})$. Let $i$ be the composition $F \rightarrow \pi_{1}(M-\Sigma) \rightarrow S$. Suppose $W$ is an $n$-cylinder one of whose boundary components is $M$. Let $G \equiv \pi_{1}(W)^{(1)}$. Then the map $j: S \longrightarrow G$ (induced by inclusion) is an algebraic $n$-solution for $i: F \rightarrow S$.

Proof. First we will establish property (1) of Definition 6.1. Since property (1) is vacuous if $n=0$, we assume $n \geq 1$. Fix an arbitrary integer $k, 0 \leq k<$ $n$. We need to consider the map $H_{1}\left(S ; \mathbb{Z} G_{k}\right) \stackrel{j_{*}}{\longrightarrow} H_{1}\left(G ; \mathbb{Z} G_{k}\right)$ induced by the inclusion map from $M$ into $W$. Let $M_{\infty}$ be the infinite cyclic cover of $M$ and 
$W_{\infty}$ be the infinite cyclic cover of $W$. First note that since $\pi_{1}\left(M_{\infty}\right)=S$ and $\pi_{1}\left(W_{\infty}\right)=G$, the map $j_{*}$ is identical to $H_{1}\left(M_{\infty} ; \mathbb{Z} G_{k}\right) \stackrel{j_{*}}{\longrightarrow} H_{1}\left(W_{\infty} ; \mathbb{Z} G_{k}\right)$. Now let $\Gamma=\pi_{1}(W) / \pi_{1}(W)_{r}^{(k+1)}$, so $\Gamma$ is PTFA. So we have the inclusion induced map of $\mathbb{Z} \Gamma$-modules $j_{*}: H_{1}(M ; \mathbb{Z} \Gamma) \longrightarrow H_{1}(W ; \mathbb{Z} \Gamma)$. But $G_{k}$ is equal to the commutator subgroup of $\Gamma$ since $H_{1}(W) \cong \mathbb{Z}$ implying that $\Gamma_{r}^{(1)}=\Gamma^{(1)}=G / G_{r}^{(k)} \equiv G_{k}$. Therefore this map can also be viewed as a map of $\mathbb{Z} G_{k}$-modules. We claim that, as a map of $\mathbb{Z} G_{k}$-modules, this is identical to our original map $j_{*}: H_{1}\left(M_{\infty} ; \mathbb{Z} G_{k}\right) \longrightarrow$ $H_{1}\left(W_{\infty} ; \mathbb{Z} G_{k}\right)$. This is because $H_{1}(M ; \mathbb{Z} \Gamma)$ is merely the first homology group of the total space of the $\Gamma$-covering space of $M$ viewed as a $\mathbb{Z} \Gamma$-module, and this total space is the same as the total space of the $\Gamma^{(1)}$-covering space of $M_{\infty}$. Thus, as $Z \Gamma^{(1)}$-modules, $H_{1}(M ; \mathbb{Z} \Gamma)$ is the same as $H_{1}\left(M_{\infty} ; \mathbb{Z} \Gamma^{(1)}\right)$. Hence we are reduced to studying $j_{*}: H_{1}(M ; \mathbb{Z} \Gamma) \longrightarrow H_{1}(W ; \mathbb{Z} \Gamma)$ as a map of $\mathbb{Z} G_{k^{-}}$-modules and we need to show that it is nontrivial, even after tensoring with $\mathbb{K}\left(G_{k}\right)$, i.e. after localizing with respect to the set $R=\mathbb{Z} \Gamma^{(1)}-\{0\}$. Since $\mathbb{Z} \Gamma$ is an Ore Domain, $R^{-1} \mathbb{Z} \Gamma$ is a flat $\mathbb{Z} \Gamma$-module, and so we just need to show that the map $j_{*}: H_{1}\left(M ; R^{-1} \mathbb{Z} \Gamma\right) \longrightarrow$ $H_{1}\left(W ; R^{-1} \mathbb{Z} \Gamma\right)$ is nontrivial as a map of $\mathbb{K}\left(G_{k}\right)$-modules. For the remainder of this proof, let $\mathbb{K}$ be the quotient field of $\mathbb{Z} \Gamma^{(1)}=\mathbb{Z} G_{k}$, which is what we have called $\mathbb{K}\left(G_{k}\right)$. Finally, note that, since $H_{1}(\Gamma) \cong \mathbb{Z}, \mathbb{Z} \Gamma$ can be identified with the twisted Laurent polynomial ring $\mathbb{Z} \Gamma^{(1)}\left[t^{ \pm 1}\right]$ and, similarly, the localized ring $R^{-1} \mathbb{Z} \Gamma$ can be identified with $\mathbb{K}\left[t^{ \pm 1}\right]$. In summary, we are reduced to studying $j_{*}: H_{1}\left(M ; \mathbb{K}\left[t^{ \pm 1}\right]\right) \longrightarrow H_{1}\left(W ; \mathbb{K}\left[t^{ \pm 1}\right]\right)$ as a map of $\mathbb{K}$-modules, and we seek to show that it is nontrivial.

We now claim that Theorem 3.8 applies to the map $j_{*}: H_{1}\left(M ; \mathbb{K}\left[t^{ \pm 1}\right]\right) \longrightarrow$ $H_{1}\left(W ; \mathbb{K}\left[t^{ \pm 1}\right]\right)$. Observe that $\Gamma^{n}=0$ since $\Gamma^{k+1}=\{e\}$ and, since $k+1 \leq n$, $\Gamma^{n}=\{e\}$. Thus $\Gamma$ is an $(n-1)$-solvable PTFA group as required. We conclude that the $\mathbb{K}$-rank of $j_{*}: H_{1}\left(M ; \mathbb{K}\left[t^{ \pm 1}\right]\right) \longrightarrow H_{1}\left(W ; \mathbb{K}\left[t^{ \pm 1}\right]\right)$ is at least $(d-2) / 2$ if $n>1$ and is precisely $d / 2$ if $n=1$, where $d \equiv \operatorname{rk}_{\mathbb{Q}} H_{1}\left(M_{\infty} ; \mathbb{Q}\right)$. It is well known that $d$ is equal to the degree of the classical Alexander polynomial of $K$, which is, by hypothesis, at least 4 (or, if $n=1$ at least 2). Hence in all cases the above rank is positive, and therefore we have shown that our original map, $H_{1}\left(S ; \mathbb{Z} G_{k}\right) \stackrel{j_{*}}{\longrightarrow} H_{1}\left(G ; \mathbb{Z} G_{k}\right)$, is nontrivial even after tensoring with $\mathbb{K}\left(G_{k}\right)$.

Since the kernel of $G_{r}^{(k)} /\left[G_{r}^{(k)}, G_{r}^{(k)}\right] \longrightarrow G_{r}^{(k)} / G_{r}^{(k+1)}$ is $\mathbb{Z}$-torsion, this map is an isomorphism after tensoring with $\mathbb{K}\left(G_{k}\right)$ (which contains $\mathbb{Q}$ ). Combining this with our previous conclusion, we see that $j$ satisfies property (1) of the definition of an algebraic $n$-solution.

Now we establish property (2) of an algebraic $n$-solution. We consider an arbitrary $k$ with $0 \leq k \leq n$. By flatness, what we need to establish is the surjectivity of $i_{*}: H_{1}(F ; \mathbb{K}) \longrightarrow H_{1}(S ; \mathbb{K})$ (recall we are sometimes abbreviating $\mathbb{K}\left(G_{k}\right)$ by $\mathbb{K}$ ). Let $Y=M-\Sigma$ and $W$ be a wedge of $2 g$ circles. By choice of $f$ there is a continuous map $W \rightarrow Y$ inducing $F \rightarrow \pi_{1}(Y)$ that is 1-connected on rational homology. It follows from [COT1, Proposition 2.10] that this map induces a 1-connected map on homology with $\mathbb{K}\left(G_{k}\right)$ coefficients for any PTFA group $G_{k}$. Hence it is surjective. Since the map $i_{*}$ factors through $H_{1}(Y ; \mathbb{K})$, it suffices to prove that the map $H_{1}(Y ; \mathbb{K}) \rightarrow H_{1}(S ; \mathbb{K})=H_{1}\left(M_{\infty} ; \mathbb{K}\right)$ is surjective. If $S$ were finitely generated, this would follow as above from [COT1, Proposition 2.10]. In other words, for finitely generated groups $S$ property (2) follows from just knowing property (2) for the base case $k=0$. Unfortunately this proposition fails for general non-finitely generated 
groups and, for us, $S$ will not be finitely generated unless $K$ is fibered. Also note that the rank of $H_{1}\left(M_{\infty} ; \mathbb{K}\right)$ may well increase as the integer $k$ increases. Thus, to have a hope of establishing property $(2)$, the rank of $H_{1}(Y ; \mathbb{K})$ (which, since $Y$ has the homotopy type of a finite 2-complex, is bounded above, independently of $k$, by $2 g$ [COT1, Prop. 2.10 and 2.11]) must be a universal upper bound for the ranks of $H_{1}\left(M_{\infty} ; \mathbb{K}\right)$ for any $k$ and any coefficient system. Fortunately, the technology to establish this "universal" upper bound essentially already exists due to work of Shelly Harvey. We indicate how her work indeed establishes the result we need. For intuition, first consider the case $k=0$ where $G_{k}=0$ and $\mathbb{K}=\mathbb{Q}$. Then the result we claim is that any finite generating set for the vector space $H_{1}(Y ; \mathbb{Q})$ generates the Alexander module of $K$ (or $M$ ) as a rational vector space (a well-known result in classical knot theory). We proceed with the proof of the general case. We observed above that, as $\mathbb{Z} G_{k}$-modules, $H_{1}(M ; \mathbb{Z} \Gamma)$ is the same as $H_{1}\left(M_{\infty} ; \mathbb{Z} G_{k}\right)$. Thus, as $\mathbb{K}$-modules, $H_{1}\left(M ; \mathbb{K}\left[t^{ \pm 1}\right]\right)$ is $H_{1}\left(M_{\infty} ; \mathbb{K}\right)$ (see also the discussion above Definition 5.6). In [Ha, Proposition 7.4] (see also [C, section 6]), Harvey shows that $H_{1}\left(M ; \mathbb{K}\left[t^{ \pm 1}\right]\right)$ has a square presentation matrix (as a $\mathbb{K}\left[t^{ \pm 1}\right]$-module) whose entries are polynomials of degree at most 1 , with respect to generators that are the images under inclusion of an arbitrary generating set for $H_{1}\left(Y ; \mathbb{K}\left[t^{ \pm 1}\right]\right)$. The latter is a free module isomorphic to $H_{1}(Y ; \mathbb{K}) \otimes_{\mathbb{K}} \mathbb{K}\left[t^{ \pm 1}\right]$ (see the discussion in [Ha, section 7$])$. Choose a generating set $\left\{e_{1}, \ldots, e_{m}\right\}$ for $H_{1}(Y ; \mathbb{K})$ as a $\mathbb{K}$-vector space. Then use the set $\left\{e_{i} \otimes 1\right\}$ as a $\mathbb{K}\left[t^{ \pm 1}\right]$ generating set for the module $H_{1}\left(Y ; \mathbb{K}\left[t^{ \pm 1}\right]\right)$. Harvey's presentation result shows that these elements generate $H_{1}\left(M ; \mathbb{K}\left[t^{ \pm 1}\right]\right)$ as $a \mathbb{K}\left[t^{ \pm 1}\right]$-module. We claim that a closer analysis of some other work of Harvey shows the stronger fact that these actually generate as a $\mathbb{K}$-module! This will finish the verification that the map $H_{1}(Y ; \mathbb{K}) \rightarrow H_{1}\left(M_{\infty} ; \mathbb{K}\right)$ is surjective and thus finish the verification of property (2). In [Ha, Proposition 9.1], Harvey shows that any such matrix as above (whose entries are polynomials of degree at most 1) presents a module of rank $m$ over $\mathbb{K}$. It is only necessary to examine her proof carefully to see that this stronger statement is true: The given set of $m$ generators is a $\mathbb{K}$-generating set for the module. Harvey's proof involves changing the presentation matrix by certain allowable matrix operations and finally arriving at a simple matrix which she explicitly shows satisfies this stronger statement. Therefore it is only necessary to check that all the matrix operations she uses do in fact preserve the veracity of this statement. For example, clearly $\left\{x_{1}, \ldots, x_{m}\right\}$ is a $\mathbb{K}$-generating set for the (right) module if and only if $\left\{x_{1}-\left(x_{2}\right) k, x_{2}, \ldots, x_{m}\right\}$ is also, where $k$ is any nonzero element of $\mathbb{K}$. This translates into the fact that adding a nonzero left $\mathbb{K}$-multiple of a row of Harvey's matrix to another row is an allowable operation for our purposes. The key point is to not allow a $\mathbb{K}\left[t^{ \pm 1}\right]$-multiple. In fact the only matrix operation from Harvey's list that could lead to problems is: add to any row a left $\mathbb{K}\left[t^{ \pm 1}\right]$-linear combination of the other rows (because this corresponds to a change of generators). One only needs to notice that, in fact, in her proof she never uses the full generality of this operation. She only adds to any row a left multiple of another row by a nonzero element of $\mathbb{K}$ (not $\mathbb{K}\left[t^{ \pm 1}\right]$ ). This completes the proof.

The following theorem greatly generalizes [CT, Theorem 6.3] because our definition of an algebraic $n$-solution is much more general. Our proof follows the proof of [CT, Theorem 6.3] closely. In doing so we had to make decisions about what to include and what to reference. Because the result is already (logically) extremely demanding on the reader, for the reader's convenience, we have erred on the side 
of duplicating material and have included a complete proof. In addition, our proof is simpler in certain places.

Theorem 6.4. Suppose we are given $F \stackrel{i}{\longrightarrow} S$ as in Definition 6.1 . For each $n \geq 0$ there is a finite collection $\mathcal{P}_{n}$ of sets consisting of $2 g-1$, if $n>0$, or $2 g$, if $n=0$, elements of $F^{(n)}$ (we refer to such a set as a tuple even though it is unordered), with the following property: For any algebraic n-solution $r$ for $F \stackrel{i}{\longrightarrow} S$, at least one such tuple (which will be called a special tuple for $r$ ) maps to a generating set under the composition:

$F^{(n)} \longrightarrow S^{(n)} / S^{(n+1)} \cong H_{1}\left(S ; \mathbb{Z} S_{n}\right) \stackrel{r_{*}}{\longrightarrow} H_{1}\left(S ; \mathbb{Z} G_{n}\right) \longrightarrow H_{1}\left(S ; \mathbb{Z} G_{n}\right) \otimes_{\mathbb{Z} G_{n}} \mathbb{K}\left(G_{n}\right)$

where $\mathbb{K}\left(G_{n}\right)$ is the skew quotient field of fractions of $\mathbb{Z} G_{n}$.

Proof. We remark that to be used for the proof of the main theorem, it is very important to define the collections $\mathcal{P}_{n}$ so as to depend only on the knot $K$. In particular, they must not depend on the existence of any particular $n$-cylinder.

Let $\left\{x_{1}, \ldots, x_{2 g}\right\}$ be a generating set of the free group $F$. Set $\mathcal{P}_{0} \equiv\left\{\left\{x_{1}, \ldots, x_{2 g}\right\}\right\}$, the collection consisting of a single $2 g$-tuple. Set $\mathcal{P}_{1} \equiv\left\{\left\{\left[x_{i}, x_{1}\right], \ldots,\left[x_{i}, x_{i-1}\right]\right.\right.$, $\left.\left.\left[x_{i}, x_{i+1}\right], \ldots,\left[x_{i}, x_{2 g}\right]\right\} \mid 1 \leq i \leq 2 g\right\}$, the collection consisting of $(2 g-1) \cdot(2 g)$ number of $(2 g-1)$-tuples. Supposing $\mathcal{P}_{k}(k \geq 1)$ has been defined, define $\mathcal{P}_{k+1}$ recursively as follows. For each $\left\{w_{1}, \ldots, w_{2 g-1}\right\} \in \mathcal{P}_{k}$ include the $(2 g-1)$-tuple $\left\{z_{1}, \ldots, z_{2 g-1}\right\}$ in $\mathcal{P}_{k+1}$ if $z_{i}=\left[w_{i}, w_{i}^{x_{j}}\right](1 \leq i \leq 2 g-1,1 \leq j \leq 2 g$, and $i \neq j)$ or if $z_{i}=\left[w_{i}, w_{k}\right]$ for some $1 \leq i, k \leq 2 g-1$ and $i \neq k$. Here $w_{i}^{x_{j}} \equiv x_{j}^{-1} w_{i} x_{j}$. Clearly $z_{i} \in F^{(k+1)}$.

Now we fix $n$ and show $\mathcal{P}_{n}$ satisfies the conditions of the theorem. Fix an algebraic $n$-solution $r: S \longrightarrow G$. We must show that there exists a special tuple in $\mathcal{P}_{n}$ corresponding to $r$. This is trivially true for $n=0$ using property (2) of the definition of an algebraic $n$-solution, so we assume $n \geq 1$. Now we need some preliminary definitions.

Recall that $F$ is the free group on $\left\{x_{1}, \ldots, x_{2 g}\right\}$. Its classifying space has a standard cell structure as a wedge of $2 g$ circles $W$. Our convention is to consider its universal cover $\widetilde{W}$ as a right $F$-space as follows. Choose a preimage of the 0 -cell as basepoint denoted $*$. For each element $w \in F \equiv \pi_{1}(W)$, lift $w^{-1}$ to a path $\left(\tilde{w}^{-1}\right)$ beginning at $*$. There is a unique deck translation $\Phi(w)$ of $\widetilde{W}$ which sends * to the endpoint of this lift. Then $w$ acts on $\widetilde{W}$ by $\Phi(w)$. This is the conjugate action of the usual left action as in [Ma]. Taking the induced cell structure on $\widetilde{W}$ and tensoring with an arbitrary left $\mathbb{Z} F$-module $A$ gives an exact sequence

$$
0 \longrightarrow H_{1}(F ; A) \stackrel{d}{\longrightarrow} A^{2 g} \longrightarrow A \longrightarrow H_{0}(F ; A) \longrightarrow 0 .
$$

Specifically consider $A=\mathbb{Z} G$ where $\mathbb{Z} F$ acts by left multiplication via a homomorphism $\phi: F \longrightarrow G$. From the interpretation of $H_{1}(F ; \mathbb{Z} G)$ as $H_{1}$ of a $G$-cover of $W$, one sees that an element $g$ of $\operatorname{Ker}(\phi)$ can be considered as an element of $H_{1}(F ; \mathbb{Z} G)$. We claim that the composition $\operatorname{Ker}(\phi) \longrightarrow H_{1}(F ; \mathbb{Z} G) \stackrel{d}{\longrightarrow}(\mathbb{Z} G)^{2 g}$ can be calculated using the "free differential calculus" $\partial=\left(\partial_{1}, \ldots, \partial_{2 g}\right)$ where $\partial_{i}: F \longrightarrow \mathbb{Z} F$. 
Specifically we assert that the diagram below commutes:

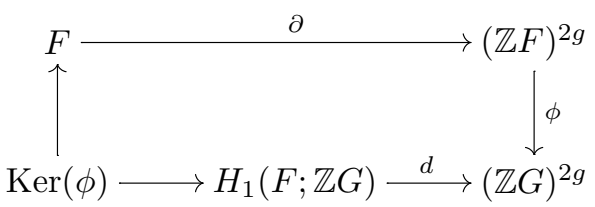

where $\partial_{i}\left(x_{j}\right)=\delta_{i j}, \partial_{i}(e)=0$ and $\partial_{i}(g h)=\partial_{i} g+\left(\partial_{i} h\right) g^{-1}$ for each $1 \leq i \leq 2 g$. Note that the usual formula for the standard left action is $d_{i}(g h)=d_{i}(g)+g d_{i}(h)$. Our formula is obtained by setting $\partial_{i}=\bar{d}_{i}$ where ${ }^{-}$is the involution on the group ring. This is justified in more detail in [CT, Section 6].

Henceforth we abbreviate maps of the form $(r, \ldots, r):\left(\mathbb{Z} F_{n}\right)^{2 g} \longrightarrow\left(\mathbb{Z} G_{n}\right)^{2 g}$ as $r$. Note that $r \circ \pi_{k}: F \rightarrow F_{k} \rightarrow G_{k}$ is the same as $\pi_{k} \circ r: F \rightarrow S \rightarrow G \rightarrow G_{k}$.

Lemma 6.5. Given an algebraic n-solution $r: S \longrightarrow G$ for $F \rightarrow S$, for each $k$, $1 \leq k \leq n$ there is an ordering of the basis elements $\left\{x_{1}, \ldots, x_{2 g}\right\}$ of $F$ such that there exists at least one tuple $\left\{w_{1}, \ldots, w_{2 g-1}\right\} \in \mathcal{P}_{k}$ with the following good property: The set of $2 g-1$ vectors (obtained as $i$ varies from 1 to $2 g-1)\left(r \pi_{k} \partial_{1} w_{i}, \ldots, r \pi_{k} \partial_{2 g-1} w_{i}\right)$ (i.e., the vectors consisting of the first $2 g-1$ coordinates of the images of the $w_{i}$ under the composition $\left.F^{(k)} \stackrel{\pi_{k} \partial}{\longrightarrow}\left(\mathbb{Z} F_{k}\right)^{2 g} \stackrel{r}{\longrightarrow}\left(\mathbb{Z} G_{k}\right)^{2 g}\right)$ is right linearly independent over $\mathbb{Z} G_{k}$.

Proof that Lemma $6.5 \Longrightarrow$ Theorem 6.4 . The set $\mathcal{P}_{n}$ has been defined. Recall that we are assuming that $n \geq 1$. Given an algebraic $n$-solution the lemma provides a tuple $\left\{w_{1}, \ldots, w_{2 g-1}\right\} \in \mathcal{P}_{n}$ which has the good property. We verify that any good tuple $\left\{w_{1}, \ldots, w_{2 g-1}\right\}$ for $r$ is a special tuple for $r$. Consider the diagram below. Recall $G_{n}=G / G_{r}^{(n)}$.

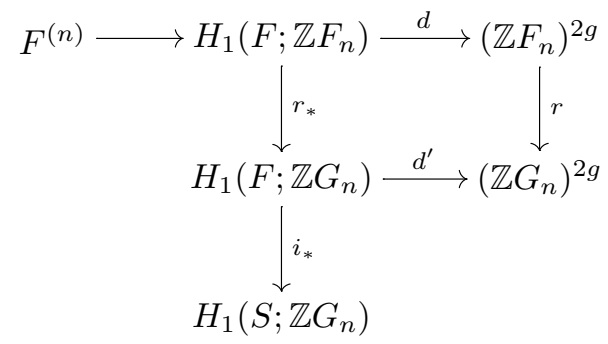

The horizontal composition on top is $\pi_{n} \circ \partial$. The right-top square commutes by naturality of the sequence (1) above. The rank of $H_{1}\left(F ; \mathbb{Z} G_{n}\right)$ over $\mathbb{K}\left(G_{n}\right)$ is $2 g-1$ by [C, Lemma 3.9]. Since $d^{\prime}$ is a monomorphism, and $i_{*}$ is an epimorphism after tensoring with $\mathbb{K}\left(G_{n}\right)$, to show $\left\{w_{1}, \ldots, w_{2 g-1}\right\}$ is special, it suffices to show that the set $\left\{r \pi_{n} \partial\left(w_{1}\right), \ldots, r \pi_{n} \partial\left(w_{2 g-1}\right)\right\}$ is $\mathbb{Z} G_{n}$-linearly independent in $\left(\mathbb{Z} G_{n}\right)^{2 g}$. This follows immediately from the good property of the tuple $\left\{w_{1}, \ldots, w_{2 g-1}\right\}$. This completes the verification that the lemma implies the theorem.

Proof of Lemma 6.5. The integer $n$ is fixed throughout. Let $r: S \rightarrow G$ be a fixed algebraic $n$-solution. Recall that, by Remark 6.2(1), for any ordering of the basis elements of $F$, there is some $i$ such that $r_{*}\left(x_{i}\right)$ is nontrivial in $G_{1}=G / G_{r}^{(1)}$. Thus by reordering we may assume that $r_{*}\left(x_{2 g}\right)$ is nontrivial in $H_{1}(G ; \mathbb{Q})=G_{1} \otimes \mathbb{Q}$. Since $G_{1} \equiv G / G_{r}^{(1)}$ is torsion-free, this means that $r \pi_{1}\left(x_{2 g}\right)$ is nontrivial in $G_{1}$. 
We will prove the lemma by induction on $k$. We begin with $k=1$. Consider the $(2 g-1)$-tuple $\left\{z_{1}, \ldots, z_{2 g-1}\right\}=\left\{\left[x_{2 g}, x_{1}\right], \ldots,\left[x_{2 g}, x_{2 g-1}\right]\right\} \in \mathcal{P}_{1}$. We claim that it has the good property. Using $d$ as shorthand for $\partial_{j}$, one computes that $d([g, h])=$ $d g+(d h) g^{-1}-(d g) g h^{-1} g^{-1}-(d h) h g h^{-1} g^{-1}$. Using this we compute that, for any $j$, $1 \leq j \leq 2 g-1, \partial_{j} z_{i}$ is $x_{2 g}^{-1}-\left[x_{i}, x_{2 g}\right]$ if $j=i$ and is otherwise zero. Thus, only the $i^{t h}$ coordinate of $\left(r \pi_{k} \partial_{1} z_{i}, \ldots, r \pi_{k} \partial_{2 g-1} z_{i}\right)$ is (possibly) nonzero. Therefore the square matrix whose columns are these vectors is a diagonal matrix and to establish the good property, it suffices to show that $r \pi_{1}\left(x_{2 g}^{-1}-\left[x_{i}, x_{2 g}\right]\right) \neq 0$ since this certainly implies that $r \pi_{k}\left(x_{2 g}^{-1}-\left[x_{i}, x_{2 g}\right]\right) \neq 0$. This follows since $r \pi_{1}\left(\left[x_{i}, x_{2 g}\right]\right)=e$ and $r \pi_{1}\left(x_{2 g}^{-1}\right)$ is nontrivial by assumption. Therefore the base of the induction $(k=1)$ is established.

Now suppose the conclusions of the lemma have been established for $1, \ldots, k$ where $k<n$. We establish them for $k+1$. Note that $r: S \rightarrow G$ is also an algebraic $k$-solution for $i: F \rightarrow S$. By induction the lemma holds for $k<n$, so there is a tuple $\left\{w_{1}, \ldots, w_{2 g-1}\right\} \in \mathcal{P}_{k}$ that has the good property. First, we claim that there is at least one of the $w_{i}$ (which by relabelling we will assume is $w_{1}$ ) such that $r \pi_{k+1}\left(w_{1}\right) \neq e$ in $G_{k+1}$. For, by the proof of Lemma $6.5 \Longrightarrow$ Theorem 6.4 , we know that $\left\{w_{1}, \ldots, w_{2 g-1}\right\} \in \mathcal{P}_{k}$ is special for $r$. Thus under the composition $F^{(k)} \longrightarrow H_{1}\left(S ; \mathbb{Z} S_{k}\right) \stackrel{r_{*}}{\longrightarrow} H_{1}\left(S ; \mathbb{Z} G_{k}\right) \longrightarrow H_{1}\left(S ; \mathbb{Z} G_{k}\right) \otimes \mathbb{K}\left(G_{k}\right)$ the set $\left\{w_{i}\right\}$ maps to a generating set. Combined with the fact that $r$ is also an algebraic $n$-solution, we see that the composition of the above with the map

$$
H_{1}\left(S ; \mathbb{Z} G_{k}\right) \otimes \mathbb{K}\left(G_{k}\right) \stackrel{r_{*}}{\longrightarrow} H_{1}\left(G ; \mathbb{Z} G_{k}\right) \otimes \mathbb{K}\left(G_{k}\right) \cong G_{r}^{(k)} / G_{r}^{(k+1)} \otimes \mathbb{K}\left(G_{k}\right)
$$

is nontrivial when restricted to $\left\{w_{i}\right\}$. On the other hand the combined map $F^{(k)} \longrightarrow$ $G_{r}^{(k)} / G_{r}^{(k+1)}$ is clearly given by $w_{i} \mapsto r \pi_{k+1}\left(w_{i}\right)$, so it is not possible that all of the elements $r \pi_{k+1}\left(w_{i}\right)$ lie in $G_{r}^{(k+1)}$. Hence we may assume that $r \pi_{k+1}\left(w_{1}\right) \neq e$ in $G_{k+1}$.

Consider the tuple $\left\{z_{1}, \ldots, z_{2 g-1}\right\} \in \mathcal{P}_{k+1}$, where $z_{i}=\left[w_{i}, w_{i}^{x_{2 g}}\right]$ if $r \pi_{k+1}\left(w_{i}\right) \neq e$ in $G_{k+1}$, and $z_{i}=\left[w_{i}, w_{1}\right]$ if $r \pi_{k+1}\left(w_{i}\right)=e$ in $G_{k+1}$. We will show that this tuple has the good property, finishing the inductive proof of Lemma 6.5.

For the remainder of this proof we write $x$ for $x_{2 g}$, suppressing the subscript. We need to show that the set of $2 g-1$ vectors (obtained as $i$ varies from 1 to $2 g-1$ ) $\left(r \pi_{k+1} \partial_{1} z_{i}, \ldots, r \pi_{k+1} \partial_{2 g-1} z_{i}\right)$ is $\mathbb{Z} G_{k+1}$-linearly independent. For this purpose we compute, for each $i, \partial_{j} z_{i}$. This computation falls into two cases.

Case 1. $r \pi_{k+1}\left(w_{i}\right) \neq e$.

Let $d$ be shorthand for $\partial_{j}$ for $1 \leq j \leq 2 g-1$. One has $d x=0, d\left(g^{-1}\right)=$ $(d g) g$, and $d g^{x}=(d g) x$. Using these one computes that $d\left(\left[w_{i}, w_{i}^{x}\right]\right)=\left(d w_{i}\right) p_{i}$ where $p_{i}$ is independent of $j$ and is equal to $1+x w_{i}^{-1}-\left(w_{i}^{x}\right)^{-1}\left[w_{i}^{x}, w_{i}\right]-x\left[w_{i}^{x}, w_{i}\right]$. Thus for any value of $i$ that falls under Case 1 , the vector $\left(\partial_{1} z_{i}, \ldots, \partial_{2 g-1} z_{i}\right)$ is a right multiple of the vector $\left(\partial_{1} w_{i}, \ldots, \partial_{2 g-1} w_{i}\right)$ by the element $p_{i}$. Hence the vector in question, $\left(r \pi_{k+1} \partial_{1} z_{i}, \ldots, r \pi_{k+1} \partial_{2 g-1} z_{i}\right)$, is a right multiple of the vector $\left(r \pi_{k+1} \partial_{1} w_{i}, \ldots, r \pi_{k+1} \partial_{2 g-1} w_{i}\right)$ by the element $r \pi_{k+1} p_{i}$ which lies in $\mathbb{Z} G_{k+1}$. The right factor $r \pi_{k+1} p_{i}$ is seen to be nontrivial in $\mathbb{Z} G_{k+1}$ as follows. Note $r \pi_{k+1} p_{i}$ is a linear combination of 4 group elements $e, r \pi_{k+1}\left(x w_{i}^{-1}\right), r \pi_{k+1}\left(\left(w_{i}^{x}\right)^{-1}\right)$, and $r \pi_{k+1}(x)$ in $G_{k+1}$. For $r \pi_{k+1} p_{i}$ to vanish in $\mathbb{Z} G_{k+1}$ the group elements would have to pair up in a precise way and in particular in such a way that $r \pi_{k+1}(x)=$ $r \pi_{k+1}\left(x w_{i}^{-1}\right)$ in $G_{k+1}$. This is a contradiction since $r \pi_{k+1}\left(w_{i}\right) \neq e$ by hypothesis. 
No other pairing is possible because the projections of the 4 elements to $G_{1}$ are $e, r \pi_{1}(x), e$ and $r \pi_{1}(x)$ and we have already noted that $r \pi_{1}(x)=r \pi_{1}\left(x_{2 g}\right)$ is nontrivial in $G_{1}$. Since these right factors are nontrivial and since $\mathbb{Z} G_{k+1}$ has no zero divisors, the right linear independence of the collection of vectors obtained by ignoring the $p_{i},\left(r \pi_{k+1} \partial_{1} w_{i}, \ldots, r \pi_{k+1} \partial_{2 g-1} w_{i}\right)$, would be sufficient to imply the right linear independence of the original set of vectors. We denote these new vectors by $\mathbf{v}_{\mathbf{i}}^{\mathbf{k}+\mathbf{1}}$. So far we have only dealt with those values of $i$ that fall under Case 1 .

Case 2. $r \pi_{k+1}\left(w_{i}\right)=e$.

As above one computes that $d\left(\left[w_{i}, w_{1}\right]\right)=\left(d w_{i}\right) q_{i}+\left(d w_{1}\right)\left(w_{i}^{-1}-\left[w_{1}, w_{i}\right]\right)$ where $q_{i}$ is independent of $j$ and is equal to $1-w_{1}^{-1}\left[w_{1}, w_{i}\right]$. Note that under the map $r \pi_{k+1}$ the factor $\left(w_{i}^{-1}-\left[w_{1}, w_{i}\right]\right)$ goes to zero. Thus for any value of $i$ that falls under Case 2 , the vector in question, $\left(r \pi_{k+1} \partial_{1} z_{i}, \ldots, r \pi_{k+1} \partial_{2 g-1} z_{i}\right)$, is a right multiple of the vector $\left(r \pi_{k+1} \partial_{1} w_{i}, \ldots, r \pi_{k+1} \partial_{2 g-1} w_{i}\right)$ by the element $r \pi_{k+1} q_{i}$. We denote the latter vector by $\mathbf{v}_{\mathbf{i}}^{\mathbf{k}+\mathbf{1}}$ as above. An argument just as in Case 1 shows that the right factor $r \pi_{k+1} q_{i}$ is nontrivial, using the nontriviality of $r \pi_{k+1}\left(w_{1}\right)$.

Now our objective is to show that the set of vectors $\mathbf{v}_{\mathbf{i}}^{\mathbf{k}+\mathbf{1}}$ is $\mathbb{Z} G_{k+1}$-linearly independent. Recall that our hypothesis is that the set of vectors $\left(r \pi_{k} \partial_{1} w_{i}, \ldots\right.$, $\left.r \pi_{k} \partial_{2 g-1} w_{i}\right)$, which we denote by $\mathbf{v}_{\mathbf{i}}^{\mathbf{k}}$, is $\mathbb{Z} G_{k}$-linearly independent. Note that, for each $i, \mathbf{v}_{\mathbf{i}}^{\mathbf{k}}$ is the image of $\mathbf{v}_{\mathbf{i}}^{\mathbf{k}+\mathbf{1}}$ under the canonical projection $\left(\mathbb{Z} G_{k+1}\right)^{2 g-1}$

$\left(\mathbb{Z} G_{k}\right)^{2 g-1}$. We assert that the linear independence of $\mathbf{v}_{\mathbf{i}}^{\mathbf{k}}$ implies the linear independence of $\mathbf{v}_{\mathbf{i}}^{\mathbf{k}+\mathbf{1}}$ since the kernel of $G_{k+1} \longrightarrow G_{k}$ is a torsion free abelian group and hence a $D(\mathbf{Z})$-group in the sense of R. Strebel. Details follow. This will complete the verification that $\left\{z_{1}, \ldots, z_{2 g-1}\right\}$ has the good property.

To establish our assertion above, consider that the vectors $\mathbf{v}_{\mathbf{i}}^{\mathbf{k}+\mathbf{1}}$ describe an endomorphism $f$ of free right $\mathbb{Z} G_{k+1}$-modules $f:\left(\mathbb{Z} G_{k+1}\right)^{2 g-1} \longrightarrow\left(\mathbb{Z} G_{k+1}\right)^{2 g-1}$ given by multiplying on the left by the matrix $M$ whose columns are the $\mathbf{v}_{\mathbf{i}}^{\mathbf{k}+\mathbf{1}}$. Then $f$ induces such a map $\bar{f}:\left(\mathbb{Z} G_{k}\right)^{2 g-1} \longrightarrow\left(\mathbb{Z} G_{k}\right)^{2 g-1}$ given by the matrix $\bar{M}$ obtained by projecting each entry of $M$. Thus the columns are the $\mathbf{v}_{\mathbf{i}}^{\mathbf{k}}$. Let $H=G_{r}^{(k)} / G_{r}^{(k+1)}$ and note that $\mathbb{Z} G_{k+1} \otimes_{\mathbb{Z}[H]} \mathbb{Z} \cong \mathbb{Z} G_{k}$ where $a \otimes 1 \mapsto \bar{a}$, as $\mathbb{Z} G_{k+1}-\mathbb{Z}$-bimodules. Moreover (under this identification) $f$ descends to $f \otimes$ id : $\left(\mathbb{Z} G_{k}\right)^{2 g-1} \longrightarrow\left(\mathbb{Z} G_{k}\right)^{2 g-1}$ sending $\bar{v}$ (for $v \in\left(\mathbb{Z} G_{k+1}\right)^{2 g-1}$ ) to $\bar{f}(\bar{v})$, thus agreeing with $\bar{f}$ above. Our hypothesis on $\mathbf{v}_{\mathbf{i}}^{\mathbf{k}}$ guarantees that the columns of $\bar{M}$ are right linearly independent and hence that $\bar{f}$ is injective. Since $H$ is torsion-free-abelian, a theorem of Strebel [Str, Section 1] ensures that the injectivity of $f \otimes \mathrm{id}=\bar{f}$ implies the injectivity of $f$. This in turn implies the linear independence of the columns of $M$, which are the $\mathbf{v}_{\mathbf{i}}^{\mathbf{k}+\mathbf{1}}$.

Now we give a proof of Theorem 5.13.

Proof of Theorem 5.13. Let $S \equiv \pi_{1}(M)^{(1)}$. Suppose $W$ is an $n$-cylinder with $M$ as one of its boundary components. The inclusion $j: M \longrightarrow W$ induces a map $j: S \longrightarrow \pi_{1}(W)^{(1)}$. Let $G \equiv \pi_{1}(W)^{(1)}=\pi_{1}(W)_{r}^{(1)}$. The last equality holds since $H_{1}(W) \cong \mathbb{Z}$. For convenience, let us use the same notation $\Sigma$ for the capped-off Seifert surface. Let $g \equiv \frac{1}{2} \operatorname{rk}_{\mathbb{Q}} H_{1}(M \backslash \Sigma ; \mathbb{Q}$ ) (which is equal to the genus of $\Sigma$ ). Let $F$ be the free group of rank $2 g$. Choose a map $F \longrightarrow \pi_{1}(M \backslash \Sigma)$ that induces an isomorphism on $H_{1}(F ; \mathbb{Q})$. Let $i$ be the composition $F \longrightarrow \pi_{1}(M \backslash \Sigma) \subset S$. For a group $G$, let $G_{k}$, for $k \in \mathbb{N}_{0}$, denote $G / G_{r}^{(k)}$. By [Ha, Corollary 3.6] $G_{k}$ is 
PTFA. By [COT1, Proposition 2.5] $\mathbb{Z} G_{k}$ embeds into the (skew) quotient field of fractions, which is denoted by $\mathbb{K}\left(G_{k}\right)$. By [Ste, Proposition II.3.5], $\mathbb{K}\left(G_{k}\right)$ is flat over $G_{k}$. Since $\pi_{1}(W) / \pi_{1}(W)^{(1)} \cong \mathbb{Z}$, if we let $\Gamma_{k} \equiv \pi_{1}(W) / \pi_{1}(W)_{r}^{(k+1)}$, there is a short exact sequence $1 \rightarrow G_{k} \rightarrow \Gamma_{k} \stackrel{\pi}{\rightarrow} \mathbb{Z} \rightarrow 1$ where $\pi$ is the abelianization. Then we have a PID $\mathbb{K}\left(G_{k}\right)\left[t^{ \pm 1}\right]$ such that $\mathbb{Z} \Gamma_{k} \subset \mathbb{K}\left(G_{k}\right)\left[t^{ \pm 1}\right] \subset \mathcal{K}$ where $\mathcal{K}$ is the (skew) quotient field of fractions of $\mathbb{Z} \Gamma_{k}$ ( $\Gamma_{k}$ is PTFA by [Ha, Corollary 3.6]; hence $\mathbb{Z} \Gamma_{k}$ embeds into the skew quotient field of fractions).

Apply Theorem 6.4 to find a finite collection $\mathcal{P}_{n-1}$ of $(2 g-1)$-tuples $(2 g$ if $n=1$ ) of elements of $F^{(n-1)}$. Since $S^{(n-1)}=\pi_{1}(M)^{(n)}$, the image of the union of the elements of $\mathcal{P}_{n-1}$ under $i: F \longrightarrow S$ is a finite set $\left\{\alpha_{1}, \ldots, \alpha_{m}\right\}$ of elements of $\pi_{1}(M)^{(n)}$ as required by part (1) in the statement. By Proposition 6.3 the induced map $j: S \longrightarrow G$ is an algebraic $n$-solution, hence an algebraic $(n-1)$-solution (see Remark 6.2(3)). By our choice of $\mathcal{P}_{n-1}$ from Theorem 6.4, at least one tuple $\left\{w_{1}, w_{2}, \ldots, w_{2 g-1}\right\} \in \mathcal{P}_{n-1}\left(\left\{w_{1}, w_{2}, \ldots, w_{2 g}\right\}\right.$ if $\left.n=1\right)$ maps to a generating set of $H_{1}\left(S ; \mathbb{K}\left(G_{n-1}\right)\right)$. Notice that $H_{1}\left(S ; \mathbb{K}\left(G_{n-1}\right)\right) \cong H_{1}\left(M ; \mathbb{K}\left(G_{n-1}\right)\left[t^{ \pm 1}\right]\right)$ and $H_{1}\left(G ; \mathbb{K}\left(G_{n-1}\right)\right) \cong H_{1}\left(W ; \mathbb{K}\left(G_{n-1}\right)\left[t^{ \pm 1}\right]\right)$ as $\mathbb{K}\left(G_{n-1}\right)$-modules. By Theorem 3.8 (with $\Gamma=\pi_{1}(W) / \pi_{1}(W)_{r}^{(n)},[\Gamma, \Gamma]=\pi_{1}(W)^{(1)} / \pi_{1}(W)_{r}^{(n)}=G_{n-1}$ ), we see that at least $\frac{1}{2}(d-2)$ of $\left\{w_{1}, \ldots, w_{2 g-1}\right\}$ (if $n>1$ ) or at least $\frac{1}{2} d$ of $\left\{w_{1}, \ldots, w_{2 g}\right\}$ (if $n=1)$ map nontrivially under $F^{(n-1)} \rightarrow S^{(n-1)} \stackrel{j}{\rightarrow} G_{r}^{(n-1)} / G_{r}^{(n)}\left(\cong H_{1}\left(G ; \mathbb{Z} G_{n-1}\right)\right.$ modulo $\mathbb{Z}$-torsion). Hence at least $\frac{1}{2}(d-2)$ (if $n>1$ ) or at least $\frac{1}{2} d$ (if $n=1$ ) of the $\alpha_{i}$ have the property that $j_{*}\left(\alpha_{i}\right) \notin G_{r}^{(n)}=\left(\pi_{1}(W)\right)_{r}^{(n+1)}$. Since each $\alpha_{i}$ actually lies in $\pi_{1}\left(S^{3} \backslash \Sigma\right)^{(n)}$ we can represent the $\alpha_{i}$ by simple closed curves in the complement of the chosen Seifert surface $\Sigma$ for the knot $K$. We can alter these by crossing changes until the collection of $\alpha_{i}$ forms a trivial link in $S^{3}$. Moreover, by [CT, Lemma 3.9] and its proof, we may choose representatives in the same homotopy classes that have the much stronger property that they bound (smoothly embedded) symmetric capped gropes of height $n$, disjointly embedded in $S^{3}-K$ except for the caps. This is the collection $\left\{\eta_{i}\right\}$ required.

\section{REFERENCES}

[CS] S. Cappell and J. L. Shaneson, The codimension two placement problem and homology equivalent manifolds, Ann. of Math. (2) 99 (1974), 277-348. MR0339216 (49:3978)

[CG] A. J. Casson and C. McA. Gordon, Cobordism of classical knots, printed notes, Orsay, 1975, published in A la recherche de la topologie perdue 62, ed. Guilllou and Marin, Progress in Mathematics, 1986, pp. 181-199.

[ChG] J. Cheeger and M. Gromov, Bounds on the von Neumann dimension of $L^{2}$-cohomology and the Gauss-Bonnet theorem for open manifolds, J. Differential Geom. 21 (1985), no. 1, 1-34. MR806699 (87d:58136)

[COT1] T. D. Cochran, K. E. Orr, and P. Teichner, Knot concordance, Whitney towers and $L^{2}$-signatures, Ann. of Math. (2) 157 (2003), no. 2, 433-519. MR1973052 (2004i:57003)

[COT2] T. D. Cochran, K. E. Orr, and P. Teichner, Structure in the classical knot concordance group, Comment. Math. Helv. 79 (2004), no. 1, 105-123. MR2031301 (2004k:57005)

[C] T. Cochran, Noncommutative Knot Theory, Algebr. Geom. Topol. 4 (2004), 347-398. MR2077670 (2005k:57023)

[CT] T. D. Cochran and P. Teichner, Knot concordance and von Neumann $\eta$-invariants, Duke Math. Journal 137 (2007), no. 2, 337-379.

[F] M. H. Freedman, The topology of four dimensional manifolds, J. Differential Geom. 17 (1982), no. 3, 357-453. MR679066 (84b:57006) 
[FQ] M. H. Freedman and F. Quinn, Topology of 4-manifolds, Princeton Mathematical Series, 39, Princeton University Press, Princeton, NJ, 1990. MR1201584 (94b:57021)

[Fr] S. Friedl, Eta invariants as sliceness obstructions and their relation to Casson-Gordon invariants, Algebr. Geom. Topol. 4 (2004), 893-934. MR2100685 (2005j:57016)

[FT] S. Friedl and P. Teichner, New topologically slice knots, Geom. Topol. 9 (2005), 21292158. MR2209368 (2007b:57007)

[G] P. M. Gilmer, Slice knots in $S^{3}$, Quart. J. Math. Oxford Ser. (2) 34 (1983), no. 135, 305-322. MR711523 (85d:57004)

[Ha] S. Harvey, Higher-order polynomial invariants of 3-manifolds giving lower bounds for the Thurston norm, Topology 44 no.5 (2005) 895-945. MR2153977 (2006g:57019)

[K] T. Kim, An infinite family of non-concordant knots having the same Seifert form, Comment. Math. Helv. 80 (2005), no. 1, 147-155. MR2130571 (2006a:57007)

[KL] P. Kirk and C. Livingston, Twisted Alexander invariants, Reidemeister torsion, and Casson-Gordon invariants, Topology 38 (1999), no. 3, 635-661. MR1670420 (2000c:57010)

[L] J. Levine, Knot cobordism groups in codimension two, Comment. Math. Helv. 44 (1969), 229-244. MR0246314 (39:7618)

[Le] C. F. Letsche, An obstruction to slicing knots using the eta invariant, Math. Proc. Cambridge Philos. Soc. 128 (2000), no. 2, 301-319. MR1735303 (2001b:57017)

[Li] C. Livingston, Seifert forms and concordance, Geom. Topol. 6 (2002), 403-408. MR1928840 (2003f:57019)

[Ma] W.S. Massey, Algebraic Topology: An Introduction, Harcourt, Brace, and World, 1967. MR0211390 (35:2271)

[OS] P. Ozsváth and Z. Szabó, Knot Floer homology and the four-ball genus, Geom. Topol. 7 (2003), 615-639. MR2026543 (2004i:57036)

[R] M. Ramachandran, von Neumann index theorems for manifolds with boundary, J. Differential Geom. 38 (1993), no. 2, 315-349. MR1237487 (94j:58164)

[Ra] J. A. Rasmussen, Khovanov homology and the slice genus, preprint, 2004, arXiv:math. GT/0402131.

[Ste] B. Stenström, Rings of Quotients, Springer-Verlag, 1975, New York. MR0389953 (52:10782)

[Str] R. Strebel, Homological methods applied to the derived series of groups, Comment. Math. Helv. 49 (1974), 302-332. MR0354896 (50:7373)

[Wa] C. T. C. Wall, Surgery on Compact Manifolds, London Math. Soc. Monographs 1, Academic Press, 1970. MR0431216 (55:4217)

Department of Mathematics, Rice University, Houston, Texas 77005-1892

E-mail address: cochran@math.rice.edu

Department of Mathematics, Konkuk University, Seoul 143-701, Korea

E-mail address: tkim@konkuk.ac.kr 